$\widehat{\mathcal{G}}_{\text {http://dx.doi.org/10.3765/sp.5.3 }}^{\text {Semantics \& Pragmatics Volume 5, Article 3: 1-58, } 2012}$

\title{
The meaning of plural definites: A decision-theoretic approach*
}

\author{
Sophia A. Malamud \\ Brandeis University
}

Received 2011-11-01 / Accepted 2011-12-22 / Revisions Received 2012-01-12 / Published 2012-07-24

\begin{abstract}
This paper has two mutually motivating goals. The empirical goal is to demonstrate that definite plurals are expressions whose interpretation requires an approach that incorporates the goals of the speaker and hearer. The theoretical goal is to contribute to the development of a mathematical model of the pragmatic reasoning involved in the interpretation of underspecified utterances. I propose a decision-theoretic approach in which alternative interpretations of underspecified expressions are built up compositionally, and the resulting interpretation is the disjunction of maximally optimal alternatives. For definite plurals, this derives the correct interpretations in a variety of linguistic and situational contexts based on pragmatic and lexical cues.
\end{abstract}

Keywords: homogeneity presupposition, strongest meaning hypothesis, distributivity, maximality, decision theory, relevance, definite plurals

\section{Introduction}

Early work in formal linguistic semantics investigated the truth conditions of natural language expressions while relegating context-dependent aspects of

* In memory of Ellen F. Prince, a guiding light and an inspiration. I thank Maribel Romero and Robin Clark for constant encouragement and help in working on this material, and organisers, reviewers, and audiences at SALT 16, the LSA Institute 2007 workshop on Conversational Games and Strategic Inference, and the workshop on Games and Decisions in Pragmatics 3, and Vita Markman for their comments and questions. I am deeply grateful for the very useful comments from anonymous reviewers and editors of this journal. Any flaws remain my responsibility.

(C)2012 Sophia A. Malamud

This is an open-access article distributed under the terms of a Creative Commons NonCommercial License (creativecommons.org/licenses/by-nc/3.o). 
meaning to the study of pragmatics. More recent efforts in formal semantics and formal pragmatics have sought to integrate pragmatic factors into a more comprehensive formal theory of natural language interpretation. One line of inquiry into the semantics-formal pragmatics interface aims to formalise Gricean principles in terms of game theory and its subfield, decision theory. The central question raised by this approach is how much information is necessary and sufficient for successful communication. The resulting formalisation of optimally relevant (that is, necessary and sufficient) information can be naturally applied to the many other areas in which relevance rooted in speakers' goals affects semantic interpretation, including computation of truth-conditionally underspecified/contextually-dependent meanings and computation of conversational implicatures.

Decision- and game-theoretic frameworks for formal pragmatics have been explored in the research of Merin, van Rooij, Benz, Parikh, and others, to derive conversational implicatures (see van Rooij 2011, and references therein), and to resolve pronoun reference and scope ambiguities (Parikh 2000), and underspecified questions (van Rooij 2003). I am extending this line of research so as to permit a more thorough integration of pragmatic computations with the interpretation of definite plurals. My proposal integrates the semantic theory of Landman (1989) with a principled account of how and when the various readings arise, and improves upon earlier proposals by Schwarzschild (1991) and Brisson (1998). Specifically, it derives cases of intermediate distributivity (e.g., The veggies are too heavy for the small scale and too light for the big scale in a scenario in which a grocer needs to weigh pre-packaged baskets of veggies), a central goal in Schwarzschild (1991), as well as cases of team credit resulting in collective readings (e.g., The boys built a raft in a scenario in which some boys from the team slacked off), a central goal in Brisson (1998). Both Schwarzschild's and Brisson's theories incorrectly require at least the speaker to know the exact distribution of individuals and the identity of exceptions in order to interpret definite plurals. In fact, neither the exact distribution of veggies in baskets in The veggies are too heavy, nor the identity of the slackers in The boys built a raft needs to be known, an irrelevance naturally derived in my decision-theoretic approach.

The proposed interpretation process focuses on competing readings of underspecified sentences, building the alternatives compositionally. A pragmatic operator intervenes in this composition, selecting those alternatives which are maximally relevant to the Question Under Discussion, henceforth QUD, interpreted as a decision problem facing the hearer (and guessed by the 
The meaning of plural definites

speaker). The resulting meaning for sentences with underspecified definite plurals may remain underspecified between these optimal alternatives, capturing the intuition that speakers only need to be precise enough to address the QUD.

\subsection{The problem: The interpretational variability of definite plurals}

It has been long noted that definite NPs tend to get exhaustive (narrowexistential) interpretations in negative contexts (1a), while also favouring exhaustive (universal) readings in non-negative sentences ( $1 \mathrm{~b}$ ). I will name the pair of examples in (1) LINGUISTS.

\section{(1) LINGUISTS Plural definites}

a. Peter didn't see the linguists. (not any of the linguists)

b. Peter saw the linguists.

(all of the linguists)

The range of expressions that display this variability and the factors that play a role in determining the final interpretation remain the subject of debate. Schwarzschild (1994) notes the effect for definite plurals (1), and Lappin (1989) for plural pronouns. Löbner (2000) examines this negationrelated variability in predication in general, beyond just definites.

The Homogeneity Presupposition (HP) and the Strongest MeANing HYPOTHESIS (SMH) have been invoked as competing explanations for this variability. Researchers investigating the interpretation of definite plurals, generic bare plurals, conjunctions, donkey conditionals with proportional quantifiers, bare (generic) conditionals (Barker 1996, von Fintel 1997, Fodor 1970, Löbner 1985, 1987, Schwarzschild 1994, Szabolcsi \& Haddican 2004), among other things, have turned to the HP as the explanation for interpretive variability. Informally, the HP is a presupposition that a plural predicate applies either to all or none of the singularities comprising its plural argument. The SMH, a competing proposal, has been called upon to explain the interpretive variability in reciprocals, plurals (including conjunctions), and donkey anaphora (Breheny 2005, Dalrymple, Kanazawa, Kim, et al. 1998, Dalrymple, Kanazawa, Mchombo, et al. 1994, Krifka 1996b, Winter 2001), calling into question both the descriptive validity and the explanatory depth of the HP for these phenomena. The Strongest Meaning Hypothesis, which requires that a semantically underspecified expression yield the logically strongest of its meanings, can be thought of as the semantic version of the 
Gricean maxim of quantity (say as much as is required). As an instance of general rationality-based reasoning, it has more explanatory appeal than the HP.

Here, I concentrate on the analysis of definite plurals, and demonstrate that the variability is only indirectly related to the monotonicity of the environments in which these expressions are situated. I argue that both the $\mathrm{HP}$ and the SMH fail to account for the full range of data. Instead, I propose an improvement on the SMH, which ties the interpretation chosen for NPs to the conversational goals of the speaker and hearer. The theoretical part of the paper then develops a decision-theoretic approach to this generalisation of the SMH.

\subsection{The roadmap}

In the rest of this section I review previously noted, but under-appreciated, data demonstrating context-dependent variability in the interpretation of definite plurals, and describe a corpus study further corroborating the generalisations (§1.3). I also review the similarities between questions and definite plurals that provide the inspiration for my approach (§1.4). I then provide background information on the semantics of definite plurals (\$2). My proposal is presented in \$3. I discuss empirical and theoretical consequences in $\S 4$, before concluding in $\S 5$.

\subsection{Some under-appreciated data}

The readings indicated in (1) are no more than tendencies, easily overriden when lexical or pragmatic context favours a different interpretation (2). This has been noted before by Krifka (1996b), Szabolcsi \& Haddican (2004), and Breheny (2005), among others, but I think the gravity of this data has not been sufficiently appreciated.

(2) SAFE HOUSE (example inspired by Krifka (1996b), (10))

a. Situation: Mary has a large house with over a dozen windows in different rooms. She locks up and leaves to go on a road trip with her friend Max, forgetting to close just a few of the many windows in various rooms. A few minutes into the ride, Max says, "There is a thunderstorm coming. Is the house going to be OK?" Mary replies: 
The meaning of plural definites

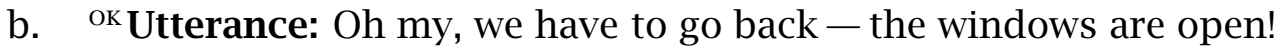

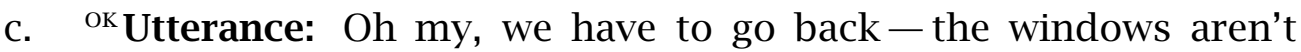
closed!

In the context in (2a), the preferred interpretation for the utterance in (2b), the example I will call SAFE HOUSE, is the opposite of the reading predicted by the HP; the same context "flips" the preference for (2c). ${ }^{1}$ Note that the non-maximality in (2b) is not a matter of domain selection: neither the open nor the exceptional closed windows represent a contextually salient subset of all the windows. It is simply irrelevant in the context how many of the windows are open, as long as some of them are.

The example in (2b) involves a bridging inference from the house to the windows. This raises the possibility that the hearer allows non-maximal (indefinite-like) interpretations of the plural definite in such contexts, because the difference between some windows and the windows here resides in the ability of the definite, and the inability of the indefinite, to trigger the bridging inference. ${ }^{2}$ The speaker's choice of the definite is then licensed because of its anaphoric function.

Variations of this example given in (3b, c), where the non-bridging definites are maximal, seem to support the possibility that non-maximality is linked to bridging. ${ }^{3}$

(3) a. Situation: as given in SAFE HOUSE. A few minutes into the ride, Max says, "There is a thunderstorm coming. Did you close the windows?"

b. Utterance: Oh my, we have to go back - the windows are open/aren't closed!

(OK maximal, ???non-maximal)

1 Ten naïve native speakers (presented with a sketch of the house in which 3 out of 10 visible windows on two different floors were open), and twelve linguists were consulted about this and other examples. Nine of the naïve native speakers and all twelve of the linguists judged SAFE HOUSE to be true and appropriate in the situation given. The same speakers, presented with a picture and a dialogue, judged two other examples with similar properties to have non-maximal, existential-like interpretations in upward-entailing contexts. On the other hand, an anonymous reviewer and the reviewer's informants rejected this example as inapproproate. For naturally occurring examples of plural definites with non-maximal, existential interpretations, see the corpus study described below.

2 I thank an anonymous reviewer for pointing out this possibility. The same reviewer also suggests the modification of SAFE HOUSE in (3), arguing that it demonstrates the inability of non-bridging definites to be non-maximal in upward-entailing contexts. In fact, the corpus study described below shows that maximality is not correlated with bridging.

3 However, note that the definite in (3c) is also a bridging one, referring to the back of the house; as a result it seems that the weakening of maximality might be tolerated here as well. 
c. Utterance: Oh my, we have to go back - the windows in the back are open/aren't closed!

(OK maximal, ???non-maximal)

In order to rule out this possible explanation, we need examples demonstrating non-maximal interpretations (in upward-entailing contexts) for nonbridging definites - both containing directly anaphoric ones, as in (3b), and uniquely identifying descriptions, as in (3c). To investigate this issue, I conducted a corpus study. All instances of definite plurals with the article the were extracted from the first half of a young-adult novel "Shatterglass", written by Tamora Pierce, a native speaker of American English, from an article by Jhumpa Lahiri, and from several conversations between native speakers of American English taken from the Michigan Corpus of Academic Spoken English, for a total of 240 examples.

All examples were judged to be grammatical, interpretable, and felicitous. The plurals were then annotated by four native speakers, each with some linguistics training. The tasks were: (i) to identify the set of entities being referred to by the definite plural (domain selection); (ii) to state the relationship of this set to linguistic context (discourse-new, bridging, anaphoric); and (iii) to determine whether the reference was maximal or non-maximal with respect to the identified set. The maximality judgement was whether "all", "some", or "not any" of the entities in the set being referred to actually participated in the predicate. To classify these interpretations according to strength and homogeneity, I also separated upward-entailing from NPI-licensing contexts.

The annotators unanimously classified 121 examples as maximal, and 46 as non-maximal. Only one non-maximal definite plural was in an NPIlicensing context, making the resulting interpretation the strongest one (though still incompatible with the homogeneity presupposition). There was no correlation between the type of definite (discourse-new, bridging, anaphoric) and its maximality. In several, but by no means all cases, the non-maximal interpretation was forced by a spatial preposition combined with world knowledge, as in (4).

(4) He ran [...] instead of dropping into a dip between the dunes. DunES In some of the other cases, and in the majority of the cases in which annotators disagreed, the main issue concerned domain selection: the context of these examples could potentially support a smaller or a larger set as the referent of the definite plural. If the annotators chose the larger set, they marked the plural as non-maximal; if the smaller set was chosen, the plural was marked as maximal. This confirms the observation that domain 
The meaning of plural definites

selection is crucial for the interpretation of definite plurals (Brisson 1998, Schwarzschild 1991). The corpus study also produced examples similar to SAFE HOUSE, where non-maximality cannot be reduced to domain selection, and does not seem to be forced lexically. One such example is given in (5) - note that the beggars is not a bridging definite, and that it is in an upward-entailing context.

\section{(5) BEgGARS}

Keth's slow speech, occasional stammer, and slight clumsiness went unnoticed in a district where the beggars were missing body parts.

Importantly, many examples with non-maximal interpretations contain non-bridging definites $(5,6)$, including uniquely identifying descriptions (6a) and those that are anaphoric (6b).

(6) a. As she walked, she looked on the sights and people of Tharios with interest. [a description of some of the sights]

b. The Tharians themselves were a feast for her eyes.

As will become clear in the discussion section (\$4), both the HP and the SMH fail to account for examples of pragmatic context-dependency like SAFE HOUSE (2) and BEGGARS $(5,6)$ : the predication in those cases is non-homogeneous (the predicate applies to some but not all of the windows/beggars/Tharians) and it is the logically weaker meaning that is preferred.

Note, however, that the goals of conversational participants play a crucial role in achieving these interpretations, since changing the goals alters the choice of interpretation, as in the example situation (7). I will refer to the utterance in (7b) as PAINTERS:

(7) PAINTERS

a. Situation: Mary has a large house with over a dozen windows in different rooms. She is about to have the window frames painted, and is preparing the house for the arrival of painters. Her friend Max says, "I think you still have a ton of work to do, right?" Mary replies:

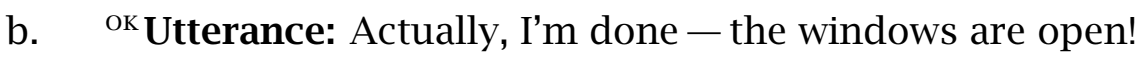

c. ? ?ок Utterance: Actually, I'm done - the windows aren't closed (anymore)! 
Although the utterances are the same, if the scenario is changed to (7a), the judgements conform to the expectations of the HP and the SMH (all of the windows are open, none are closed), in contrast to SAFE HOUSE (2).

This is not an isolated example of goal-dependent change in interpretation. Consider another situation from Krifka (1996a), in (8) below. The natural interpretation of (8b), BANK DOORS, given the arrangement of doors and the goals of the speakers, is the non-maximal one (some of the doors), going against the predictions of the SMH and the HP.

\section{(8) BANK DOORS}

a. Situation: The local bank has a safe that is accessible only through a hallway with three consecutive doors (all of which must be open to reach the safe). Alice, a teller, would like to reach the safe, and her manager says:

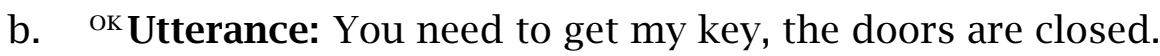

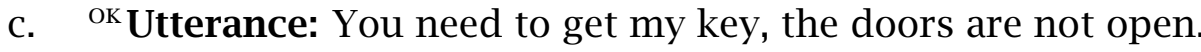

Again, changing the goals of the interlocutors reverses the judgements to the maximal interpretation (all of the doors), and brings them into alignment with the SMH and the HP. This means that the most natural interpretation in my example (9), BANK ALARM, is that all of the doors are closed.

\section{(9) BANK ALARM}

a. Situation: The local bank has a safe that is accessible through a hallway with three consecutive doors. To set the alarm when the bank closes for the night, all of the doors must be closed - if they're not, the alarm cannot be turned on. Alice, a teller, is closing up for the night, and her manager says:

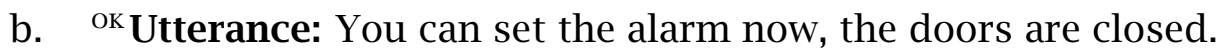

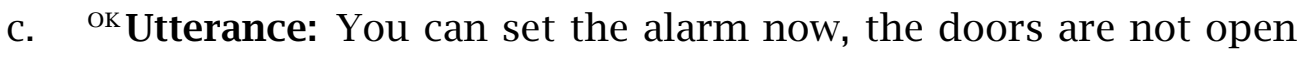
(anymore).

These examples demonstrate that interlocutors' goals play a central role in selecting the preferred interpretation. So, perhaps we can predict the readings if we take these goals into account. In the remainder of this paper, I will develop and evaluate a proposal which extends a line of research that has emerged over the last decade, seeking to incorporate pragmatic factors into a formal theory of natural language interpretation. 
The meaning of plural definites

My observations regarding the context-dependency in definite plurals are inspired by the similarity they exhibit to the context-dependent interpretation of questions. My proposal will, in fact, connect these two kinds of contextdependent expressions, building on the work of van Rooij (2003) especially. I conclude this section by reviewing the similarities between questions and plurals, and considering the contextual factors that determine interpretation.

\subsection{Underspecification in questions and plurals}

Questions are often underspecified when considered out of context; what counts as a complete answer to a question has been subject to much debate (Beck \& Rullmann 1999, Berman 1991, Groenendijk \& Stokhof 1984, Heim 1994, Lahiri 1991, inter alia).

(10) Where can I buy an Italian newspaper?

For instance, the question in (10) is underdetermined both with respect to the level of detail required in the answer (the level of granularity), and also with respect to whether all or some of the possible answers should be listed in order to create a complete answer ("mention-all" and "mention-some" interpretations of questions vary in their level of exhaustivity, cf. Beck \& Rullmann 1999). The various readings are indicated in (11).

(11) Possible readings for (10):

i. In what countries can I buy an Italian newspaper? (country-level)

ii. In what cities can I buy an Italian newspaper? (city-level)

iii. List all places selling Italian newspapers in the city. (store level, mention-all)

iv. Mention a newsstand selling Italian newspapers. (store level, mention-some)

Following Merin's (1999) application of decision theory in pragmatics, van Rooij (1999) proposes an account of question interpretation. In his theory, the level of detail and exhaustivity necessary to answer the question depends on the goals of the questioner, as (12) demonstrates.

(12) Goal 1: To gauge popularity of Italian media in Europe $\rightarrow$ reading $\mathbf{i}$ Goal 2: To see if proximity to Italy affects availability of Italian news in a European city

$\rightarrow$ reading ii

Goal 3: To write a guide for Italian visitors to the city $\rightarrow$ reading iii 
In the context of these goals, the underspecification is resolved: a single reading like the ones in (11) is chosen as the true import of the question in (10). In this way, van Rooij $(1999,2003)$ derives the level of granularity as in (11 i,ii), and degree of exhaustivity (11 iii,iv).

Striking parallels exist between the types of underspecification inherent in questions and those in definite plurals. First, as repeatedly noted in the literature, definite plurals are underspecified with respect to the level of granularity (distributivity) (13).

\section{(13) Underspecified definite plurals: distributivity $\approx$ granularity}

a. The boys built a raft.

b. Possible interpretations:

i. Team $1=\{$ Al,Bill $\}$ built a raft and Team $2=\{$ Chris,Dan $\}$ built a raft

ii. Al built a raft \& Bill built a raft \& Chris built a raft \&

Dan built a raft

Second, definites are underspecified with respect to exhaustivity: (14) is compatible with a maximal (all the windows) or non-maximal construal of the definite, e.g. excepting some closed windows in a situation like SAFE HOUSE (Krifka 1996b).

\section{(14) Underspecified definite plurals: maximality $\approx$ exhaustivity}

a. The windows are open.

b. Possible interpretations:

i. All of the windows are open

ii. Some of the windows are open

In resolving the underspecification, what factors affect the final interpretation of sentences with definite plurals? As I discuss above, the monotonicity of the context (i.e., whether it is upward- or downward-entailing) creates preferences for (non)maximality of the plurals. In addition, previous authors note that the nature of the predicate plays a role. For example, (15a) must be distributive, while (15b) must be collective (Dowty 1987). At the same time, Yoon (1996) notes that some predicates produce a preference for maximal 
The meaning of plural definites

(15c) or non-maximal (15d) interpretation. ${ }^{4}$ Spatial prepositions often force non-maximal interpretations where maximal ones would conflict with world knowledge, as in DuNES (4).

(15) a. The boys took a deep breath.

distributive

b. The boys surrounded the castle.

collective

c. The children are healthy. The windows are closed. tends maximal

d. The children are sick. The windows are open. tends non-maximal

While the predicate clearly plays a role, it does not fully determine the final interpretation - Krifka (1996b) gives BANK DOORS (8), in which closed (a total/maximal predicate, for Yoon) has a non-maximal interpretation. A point that Krifka does not make is that extra-linguistic factors play a role in resolving the underspecification in both distributivity and maximality.

(8) BANK DOORS, partially repeated

a. Situation: The local bank has a safe accessible only through a hallway with three consecutive doors (all of which must be open to reach the safe). A teller would like to reach the safe, and her manager says:

b. Utterance: You need to get my key, the doors are closed.

The contextual factors which influence the interpretation include whether there is a salient arrangement of the entities within the NP into "packages" (veggies pre-packaged in baskets, windows/doors on the outside of the house, doors in a sequence), but also, crucially, the goals of conversational participants. Schwarzschild (1991) notes the influence of packaging on distributivity with examples like (16b, VEGGIES).

(16) VEGGiEs (from Schwarzschild 1991)

a. Situation: The grocery has a big rough scale suitable only for truckloads. The owner discusses the latest delivery of vegetables, which included a truckload of them.

b. Utterance: The veggies are too light for the big scale. (excludes collective interpretation)

4 We note that these lexical preferences correlate with the semantics of the predicates: for instance, a door can be open just a bit and still count as being open (Kennedy's (2007) 'minimum standard' adjectives); for a plurality of doors this suggests a non-maximal (only some doors are open) interpretation. In contrast, a 'maximum standard' adjective like closed requires total closure, and also creates a preference for maximal interpretations. 
To demonstrate the influence of interlocutors' goals, I separate these goals and the arrangement of entities (their "packaging") from the rest of the extra-linguisic context. For example, in VEgGIES (16), a grocery-store owner who has the goal in (16d) in the situation (16a) and whose vegetables arrive as in (16c) will interpret the utterance (16b) to mean that all of the vegetables in the delivery, taken together, are too light for the big scale (the collective interpretation, which is false in this situation - the totality of delivered veggies was exactly a truckload, so suitable for the big scale).

(16) VegGies, continued

c. Packaging: The truckload of vegetables arrives at a grocery prepackaged in baskets.

d. Goal 1: To double-check the amount delivered $\rightarrow(16 \mathrm{~b})$ is False

In contrast, if the owner's goal is the one in (16e), the same packaging and situation $(16 \mathrm{a}, \mathrm{c})$ result in a different interpretation for $(16 \mathrm{~b})$ : the vegetables in each basket are too light for the big scale (the intermediate distributive interpretation, which is true in this situation).

(16) VegGies, continued

e. Goal 2: To price veggies for sale in baskets $\quad \rightarrow(16 \mathrm{~b})$ is True

The lexical and contextual influences on examples involving varying levels of maximality are similarly at play in (17) and (18). The influence of packaging the windows and doors into salient arrangements is evident in the contrast between (17) on the one hand, and (18) on the other hand. At the same time, when the packaging is kept constant, a change in the interlocutors' goals affects the maximality of the definite.

(17) SAFE HOUSE and PAINTERS: the context

a. Utterance: The doors/windows are open.

b. Situation: Mary is the owner of a large house.

c. Packaging: The house has six doors and a score of windows, on various sides along the perimeter.

d. Goal 1: Prepare for window/lintel painters $\rightarrow$ All doors/windows open

e. Goal 2: Ensure the house is thief-proof $\rightarrow$ Some doors/windows open 
The meaning of plural definites

In (17), the partial predicate open biases towards a non-maximal interpretation of the definite, as described by Yoon. However, given Goal 1, a maximal interpretation results. Keeping the contextual factors constant, note that uttering The doors/windows are closed with Goal 1 in mind results in a non-maximal interpretation - despite the fact that closed is a total predicate, the utterance is true and appropriate even if some of the doors or windows are actually open. Switching to Goal 2 changes the maximality of the definite: now, (17a) is interpreted non-maximally, while The doors/windows are closed must be maximal.

Comparing this example to (18) below, we again see that the goals of the interlocutors play a crucial role. Consider the utterance (18a) in the situation in (18b).

(18) BANK DOORS and BANK ALARM: the context

a. Utterance: The doors are closed.

b. Situation: Alice is a teller at a local bank. Her manager has keys to all the doors. To set the alarm at the end of the day, all of the internal doors leading to the safe must be closed.

c. Packaging: The bank safe is accessible only through a hallway with three consecutive doors (all of which must be open to reach the safe)

d. Goal 1: To reach the safe

$\rightarrow$ Some door(s) are closed

e. Goal 2: To set the alarm

$\rightarrow$ All doors are closed

If Alice is pursuing Goal 1 , then the utterance is true and appropriate even if not all of the doors are actually closed. Note that The doors are open in this case would be maximal. In contrast, if Alice needs to achieve Goal 2, then the interpretation is maximal for (18a), and non-maximal for The doors are open.

The data described above is what a theory of the interpretation of definite plurals needs to account for. First, such a theory needs an underspecified semantics that allows for the full range of variation in collective/distributive and maximal/non-maximal interpretations. Second, we need a way to encode the effects of the predicate, the packaging of entities within the NP denotation, and the participants' goals on the computation of truth conditions. The idea that both packaging and speaker/hearer goals play a role in determining the interpretation of definite plurals is fairly uncontroversial. Existing 
accounts, however, have either not spelled out the role of extra-linguistic factors, or focused only on the role of packaging. I detail these in the next section. My main theoretical contribution is to provide a way of including interlocutors' goals in the process by which the interpretation of a definite plural is determined.

\section{Previous approaches to the meaning of definite plurals}

In this section, I review previous work on the semantics and pragmatics of definite plurals (\$§2.1 and 2.2), culminating in a weak, underspecified semantics (\$2.3) that will serve as the foundation for pragmatic calculations that yield a contextually-appropriate interpretation. A proposal detailing the pragmatic calculations that interact with compositional semantics follows in §3.

2.1 Prior work on distributivity and maximality: Landman (1989), Link (1983)

\subsubsection{Ontology for algebraic semantics of definite plurals}

I assume denotations of the following types: objects of type $e$, eventualities of type $v$, worlds of type $s$, and truth-values of type $t$. Sentences denote propositions, which are sets of possible worlds, or, equivalently, characteristic functions of such sets.

Following the work of Link (1983), I assume that the domain of objects is partially ordered by the part-of relation, $\leq$. For instance, the substance-object denoted by the blood in my heart is part-of the object denoted by the blood in my body. Individual objects, such as the individual denoted by Ann, can also be parts of other objects, such as the plurality denoted by Ann and Bea. Such a plural expression denotes an individual sum of the two individuals $a$ and $b, a \sqcup b$. The notion of a sum generalises to multiple individuals at a time: following Sharvy (1980), the notation $\sigma x P(x)$ is used for the individual sum of all the elements in the set $P$. While a singular noun can denote a set of individuals, a plural noun can denote a set of individual sums. Semantic pluralisation is signified by the star operator, e.g., $\llbracket$ boys $\rrbracket=* b o y$ (a set of

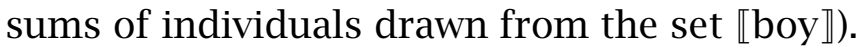

A proper part is a part which is not equal to the whole; this allows a definition of an atom as an object that has no proper parts. Unlike substances, 
The meaning of plural definites

individual objects are atomic - for instance, a chair has no proper individual parts (that is, no proper parts such that the chair is just an unstructured collection of these parts).

Alongside the concept of pure atoms, Landman (1989) introduces the notion of an impure atom, also termed a group, which can be formed from a sum by using the group-formation operation $\uparrow$. The individual parts of the sum that underlies such a plurality-turned-atom are inaccessible; to manipulate or refer to them, the operation $\downarrow$ must be used. The two operations are almost inverses of each other: $\downarrow(\uparrow(a \sqcup b))=a \sqcup b$; group-formation applied to an atom (pure or impure) yields that same atom; down-arrow applied to a sum yields that same sum. Noun phrases can shift their interpretation from sums to groups: for instance, in (19), the first predicate applies to each boy in the sum of boys, while the second predicate applies to boys as a group.

(19) The boys took a deep breath and surrounded the castle.

In a theory that recasts Schwarzschild 1991 in this framework (described in $\$ 2.3$ below), NPs only have group interpretations. The $\downarrow$ operator then becomes necessary whenever we have non-collective readings, as in (19), to turn groups into sums with accessible parts.

In summary, a sum of boys is a plurality whose individual parts (singular boys) are accessible, and can be represented as *boy or $a \sqcup b$, where $a$ and $b$ are boys. In contrast, a group of boys is a plurality whose individual parts are inaccessible, and therefore is an impure atom, represented as $\uparrow^{*}$ boy or $\uparrow(a \sqcup b)$. For instance, if Andy and Bea are individuals $a$ and $b$, then their sum is $a \sqcup b$ (e.g., the subject in Andy and Bea took a deep breath); then $\uparrow(a \sqcup b)$ is the group of Andy and Bea (e.g., if Andy and Bea are a team, the group is the subject in Andy and Bea won). Expressions $\downarrow(a \sqcup b)$ and $\downarrow \uparrow(a \sqcup b)$ both denote just the sum of Andy and Bea.

Turning to events, I follow a neo-Davidsonian view according to which verbs are predicates of events (Parsons 1990) (type $\langle v, t\rangle$ ), and sentences involve existential quantification over events. The notion of an event, often termed "eventuality", includes both eventive and stative predicates. Event participants are conceptualised not as semantic arguments of the verbs, but enter into the picture via theta-roles - functions of type $\langle v, e\rangle$. I assume a small inventory of theta-roles, such as Agent ( $\mathrm{Ag}$, the initiator of the event), Theme or Patient (Th/Pat, the undergoer of the event) etc. When generalising over theta-roles, I use the variable $\theta$ to range over the different roles. 
Following Landman (1996), among many others, I assume that events, like individual objects, form a structure of atomic (singular) events and their individual sums. Theta-roles can then serve to connect the structure of objects and the structure of events.

Singular nominal predicates, such as linguist or window denote sets of atomic (singular) objects; when pluralised, they denote sets of such atomic objects and their sums. Singular verbs denote sets of atomic events; when pluralised using the star operator, they denote a set of events and their sums. Thematic roles are only defined for atomic events, and apply to atomic individuals. As a result, to connect pluralised events and their participants, plural theta-roles are defined as in (20).

(20) Definition: * $*(e)=a$, where $a=a_{1} \sqcup a_{2} \ldots \sqcup a_{n}, e=e_{1} \sqcup e_{2} \ldots \sqcup e_{n}$ and $\theta\left(e_{1}\right)=a_{1}, \theta\left(e_{2}\right)=a_{2}, \ldots \theta\left(e_{n}\right)=a_{n}$

Paraphrase: "The plural agent, theme, ... of a sum of events is the sum of the agents, themes, ... of the atomic parts of that event sum." (Landman 1996).

Essentially, this means that a starred predicate is distributive over its participants: a pluralised open applied to a sum of windows states about each singular window in the sum that that window is open. For instance, took a deep breath in (19) is a pluralised predicate, and its plural agent is the sum of singular boys that are individual parts of the sum of boys denoted by the subject. In contrast, surrounded the castle in (19) is a singular predicate, which requires an atomic agent as a lexical requirement of the verb surround. This singular agent is the impure atom representing the boys as a group - the denotation of the subject shifts to a group interpretation.

\subsubsection{Landman's (1989) semantics for definite plurals}

Building on the work of Link (1983), Landman (1989) offers a semantic theory that permits varying levels of distributivity and (non)maximality. He uses the star (*) operator to pluralise VPs, creating distributive interpretations, and permits predicates to distribute over sums of individuals ( $a \sqcup b \sqcup c$ ), but not over groups $(\uparrow(a \sqcup b \sqcup c)$, i.e. the team of $a, b$, and $c)$. A singular event can apply either to pure or impure atoms; in the latter case, this is called "collective predication" and the plurality denoted by the group is assigned collective responsibility for participating in the singular event. 
The meaning of plural definites

All non-maximal interpretations are instances of collective predication, in which the plural is interpreted as a group.

Thus, the interpretations for $(17,18)$ would arise from the representations in (21), in a situation in which $d_{1}, \ldots, d_{5}$ are the doors (or windows) (after the arrow I give a simplified formula detailing a situation in which the interpretation is true). Note that the only way to force a fully maximal interpretation is to make it fully distributive as well (21b). ${ }^{5}$

a. $\llbracket \operatorname{SAFE} \operatorname{HOUSE}(17$, Goal 2$) \rrbracket=\llbracket$ The doors are open $\rrbracket=$ $\lambda w \exists e * \operatorname{open}(e, w) \& * T h(e)=\uparrow \sigma x * \operatorname{door}(x, w) \Rightarrow$ $\Rightarrow \uparrow\left(d_{1} \sqcup d_{2} \sqcup d_{3} \sqcup d_{4} \sqcup d_{5}\right) \in * \operatorname{open}(w)$

(collective)

b. $\llbracket$ PAINTERS $(17$, Goal 1$) \rrbracket=\llbracket$ The doors are open $\rrbracket=$ $\lambda w \exists e * \operatorname{open}(e, w) \& * T h(e)=\sigma x * \operatorname{door}(x, w) \Rightarrow$ $\Rightarrow\left(d_{1} \sqcup d_{2} \sqcup d_{3} \sqcup d_{4} \sqcup d_{5}\right) \in * \operatorname{open}(w)$

(distributive)

As can be seen from (21), Landman's theory does not provide an explanation of how and when the various interpretations of definites arise, since it does not include an account of pragmatic factors. We now turn to proposals that do consider the influence of extra-linguistic factors.

\subsection{Schwarzschild (1991) and Brisson (1998) on distributivity and maxi- mality}

Schwarzschild (1991) proposes an account of distributivity that focuses on the role of packaging, represented formally as a cover of the domain of entities, defined in (22).

(22) Cover: a set of sets of entities, such that the union of the sets in the cover is the universe of discourse.

He builds a free variable over covers $\left(\operatorname{Cov}_{i}\right)$ into the denotation of VPs, which allows them to distribute up to sub-pluralities of the definite plural given by the cover. For example, the cover in (24a) yields the intermediate-

5 With respect to (21a), I am allowing here, counterintuitively, that predicates like open or take a breath can apply to impure atoms (groups), rather than distributing to pure atoms. This allows the theory in Landman $(1989,1996)$ to derive non-maximal interpretations for open by giving the windows or doors a collective responsibility for the state of being open.

If instead we say that selectional requirements of open and take a breath demand full distributivity, then neither Landman (1989) nor the theory in Landman (1996), described in §2.3 below, can derive non-maximal readings, and a pragmatic mechanism is necessary, like the one in Brisson (1998) or the one I propose below. 
distributive reading for VEGGIES $(16,23)$ from the representation in $(24 b)$, where $a, b, c, d, e$ are the veggies.

(23) Situation: The store has a big scale suitable for truckloads, and a small scale suitable only for a few veggies.

Utterance: The veggies are too heavy for the big \& too light for the small scale.

(24) a. $\left\{\left\{a_{1}, a_{2}, b_{1}, b_{2}\right\},\{c, d, e\},\{J o h n \ldots\}\right\}$

b. $\forall x: x \in \llbracket \operatorname{Cov}_{i} \rrbracket \& x \subseteq \llbracket$ the.veg' $\rrbracket$

$$
\rightarrow x \subseteq \llbracket \text { too.heavy.sc1 \& too.light.sc2’ } \rrbracket
$$

Brisson (1998) builds on Schwarzschild (1991) to permit exceptions by allowing mismatches between the distribution of individuals into the covercells and the NP denotations. Such covers are ill-fitting covers, defined in (25).

(25) A cover is ill-fitting with respect to an NP denotation if some members of NP denotation are in the same cover-cell as non-members, so no union of cells in the cover equals the NP denotation.

Unlike Landman (1989), she only derives cases in which a few salient items are exceptional, like the bathroom window (window 5) in (26). The cover in (26a) derives the interpretation for (26b) from the representation in (26c).

a. $\left\{\left\{\right.\right.$ wind $\left._{1}\right\},\left\{\right.$ wind $\left._{2}\right\},\left\{\right.$ windw $\left._{3}\right\},\left\{\right.$ windw $\left._{4}\right\},\left\{\right.$ windw $_{5}$, Mary, door $\left.\}\right\}$

b. The windows are open (but we didn't get to the bathroom window yet).

c. $\forall x: x \in \llbracket \operatorname{Cov}_{i} \rrbracket \& x \subseteq \llbracket$ the.windows' $\rightarrow \rightarrow x \subseteq \llbracket$ open' $\rrbracket$

(26) is not an existential reading like SAFE HOUSE or BANK ALARM (2b, 17 Goal 2).

There are serious drawbacks to the cover-based account. First, the final interpretation depends on the distribution of individuals into cover-cells, making non-maximality essentially a matter of narrowing the domain to a relevant set. In this, the cover-based account of Brisson (1998) inherits the problem of any theory that derives non-maximality through domain selection: it fails to derive cases in which the context fails to select a salient set of conforming singularities, or a salient set of exceptional ones $(2 b, 5,17)$.

In fact, as example SAFE HOUSE (2b, 17), repeated as (27) below, demonstrates, non-maximality is not a matter of domain selection of any kind: there 
The meaning of plural definites

is no "relevant set" of open (non-exceptional) windows, and no window is more relevant than any other - it is simply irrelevant which or how many windows are open, as long as some of them are. This problem is intrinsic to the cover-based approach to maximality, and derives incorrect interpretations for many non-maximal uses of definite plurals.

(27) SAFE HOUSE, the cover approach

a. Utterance: The windows are open.

b. Scenario: The house has a score of windows. The hearer and speaker are going on a trip and want to make sure the house is safe.

c. Cover-derived interpretation: The relevant/salient windows are open

d. Actually: Some of the windows are open (it doesn't matter which ones, and the speaker may not know which ones)

Second, the distribution of individuals into cover-cells is denoted by a free, deictic variable, whose denotation is fixed when a particular cover is made salient in preceding discourse or extra-linguistic context. This deictic nature of $\operatorname{Cov}_{i}$ requires at least the speaker to know the exact composition of cover-cells, including the special cell containing exceptions, in the same way this is required for a pronoun. This is due to the nature of deixis - in order to point to something, speakers needs to know what they are pointing to (compare with a deictic pronoun, such as this in the example below), and deixis to covers is a speaker's pointing to a particular cover. ${ }^{6}$

(28) (Pointing) This is a book.

This is strikingly contrary to fact: the speaker doesn't need to know the identity of the vegetables for the intermediate distributivity interpretation to arise in $(16,23)$, nor is it necessary to know the identity of the closed, exceptional windows for the non-maximal interpretation to obtain in SAFE HOUSE $(2 b, 17)$.

One way to maintain a deictic approach to covers while avoiding demands

6 In cases of abstract entities serving as the denotation of a deictic pronoun, intuitions are a bit blurred. As a result, it may not be immediately clear that the speaker has perfect acquaintance with the intended referent in the example below.

(i) (No pointing) This rocks!

However, I contend that even in such cases at least the speaker has a particular abstract entity in mind, e.g., the particular activity that the speaker of (i) is engaged in at the moment. 
on speaker knowledge is to intensionalise the $\operatorname{Cov}_{i}$ variable, varying the distribution of individuals into cells with each possible world compatible with what the speaker knows. As Kratzer (forthcoming) notes, "amending Schwarzschild's account of plural predication, we would want to say that plural predication depends on contextually provided cover functions, not just on contextually provided covers." However, even such intensionalised reference to covers makes incorrect predictions when no salient cover function is provided by the situation. This is the case in our core examples SAFE HOUSE (2) and BANK DOORS (8b), where no situationally salient cover function can possibly select the exceptional and non-exceptional windows and doors.

In terms of the desiderata for a theory of definites outlined in the previous section, the cover account derives (at least some of) the influence of the VP, since the $\operatorname{Cov}_{i}$ variable is part of the VP. It also captures the influence of "packaging" in scenarios in which the context provides a salient cover that yields the correct reading. It fails to provide a correct interpretation whenever a salient cover is not available, as in the example SAFE HOUSE (2). In addition, the cover-based approach still lacks a mechanism for integrating the influence of participants' goals into the analysis.

\subsection{Landman (1996) and his recasting of Schwarzschild (1991)}

In his article "Plurality", Landman (1996) recasts Schwarzschild's proposal in his own framework (what he dubs "Theory IV" of plurality). In this theory, definite plural NPs have only group interpretations, and variations in their distributivity and maximality are achieved via a mechanism of cover roles (29). ${ }^{7}$

(29) Definition: Let $\theta$ be a thematic role. Then ${ }^{c} \theta$, the cover role based on $\theta$, is the partial function from $D_{v}$ (domain of events) into $D_{e}$ (individuals) defined as:

${ }^{c} \theta(e)=a$ iff $a \in A T O M \& \sqcup(\{\downarrow d \in S U M: d \in A T(* \theta(e))\})=\downarrow a$

Paraphrase: $a$ is a singular atom or a group, and the set underlying $a$ is the union of the elements underlying atomic subparts of the plural role based on $\theta$.

The cover roles easily derive intermediate-distributive interpretations of definite plurals. For instance, the intermediate-distributive reading for

7 Note the difference between $A T O M$ - the set of all atomic individuals - and AT (something), the set of atomic subparts underlying something. Both are used below. 
The meaning of plural definites

VEGGIES $(16,23)$, repeated below, arises from the representation in (30), where $a, b, c, r, k$ are the vegetables.

(16) VEGGIES, partially repeated

a. Situation: The grocery has a big scale suitable only for truckloads. Veggies arrive pre-packaged in baskets.

b. Utterance: The veggies are too light for the big scale.

e. Goal 2: To price veggies for sale in baskets

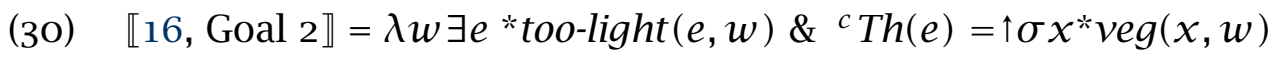
$\Rightarrow \uparrow(a \sqcup b) \sqcup \uparrow(c \sqcup r \sqcup k) \in{ }^{*} \operatorname{too}-\operatorname{light}(w)$

(intermediatedistributive)

The derivation below shows that the veggies, construed under the cover provided by the baskets, are indeed cover-themes in the event of being too light (31).

$$
\begin{aligned}
& e=f \sqcup g, T h(f)=\uparrow(a \sqcup b), T h(g)=\uparrow(c \sqcup r \sqcup k) ; \\
& \text { then *Th }(e)=\uparrow(a \sqcup b) \sqcup \uparrow(c \sqcup r \sqcup k) \\
& \{\downarrow(d): d \in A T(* T h(e))\}=\{a \sqcup b, c \sqcup r \sqcup k\} \text {, and } \\
& \sqcup(\{\downarrow(d): d \in A T(* T h(e))\})=\sqcup(\{a \sqcup b, c \sqcup r \sqcup k\}) \\
& =a \sqcup b \sqcup c \sqcup r \sqcup k= \\
& =\downarrow\left(\uparrow \sigma x^{*} \operatorname{veg}(x, w)\right)
\end{aligned}
$$

(intermediate
distributive)

q.e.d.

Cover roles are a very powerful mechanism, which derives the interpretations in $(15 a, 15 b, 17,18)$ in a similar way, as shown for $(17)$ in (32). This is unsurprising: the definition in (29) makes the plural roles used in (21) above (Landman 1989) a special case of cover roles; as a result every derivation in (21) can be done using cover roles.

(32) a. $\llbracket$ SAFE HOUSE $(17$, Goal 2$) \rrbracket=\llbracket$ The doors are open $\rrbracket$ (non-maximal, $=\lambda w \cdot \exists e^{*} \operatorname{open}(e, w) \&{ }^{c} T h(e)=\uparrow \sigma x^{*} \operatorname{door}(x, w)$ collective) $e$ is atomic, $T h(e)=\uparrow\left(d_{1} \sqcup d_{2} \sqcup d_{3} \sqcup d_{4} \sqcup d_{5}\right)$, (see footnote 5 on so $\sqcup(\{\downarrow(d): d \in A T(* T h(e))\})=\sqcup\left(\left\{d_{1}, \ldots, d_{5}\right\}\right)$ page 17) $=d_{1} \sqcup d_{2} \sqcup d_{3} \sqcup d_{4} \sqcup d_{5}=\downarrow\left(\uparrow \sigma x^{*} \operatorname{door}(x, w)\right)$

q.e.d.

b. $\llbracket$ PAINTERs $(17$, Goal 1)》= $\llbracket$ The doors are open $\rrbracket$ $=\lambda w . \exists e^{*} \operatorname{open}(e, w) \&^{c} T h(e)=\sigma x^{*} \operatorname{door}(x, w)$ (maximal, $e=f \sqcup g \ldots \sqcup j, T h(f)=d_{1}, \ldots T h(j)=d_{5}$, so *Th $(e)=\left(d_{1} \sqcup d_{2} \sqcup d_{3} \sqcup d_{4} \sqcup d_{5}\right)$ so $\sqcup(\{\downarrow(d): d \in A T(* T h(e))\})=\sqcup\left(\left\{d_{1}, \ldots, d_{5}\right\}\right)$ $=d_{1} \sqcup d_{2} \sqcup d_{3} \sqcup d_{4} \sqcup d_{5}=\downarrow\left(\uparrow \sigma x^{*} \operatorname{door}(x, w)\right)$ q.e.d. 
Take the collectively interpreted (32a), where the (trivially pluralised) VP are open in fact denotes a single atomic event. Its theme is the doors taken as a single group (impure atom) - a collective reading. Therefore, when taken down to pure singularities (individual doors), the set underlying this theme is the union of all the atomic subparts of all the themes of being-open events. As it happens, since there is only one being-open event, the derivation essentially says that when you take the set of doors underlying the theme of this event, you get the union of all sets of doors.

Landman's reinterpretation preserves the flexibility of Schwarzschild's theory, allowing for the intermediate-distributive interpretations as in VEGGIES $(16,23)$. Representations no longer contain the free, deictic variable $\operatorname{Cov}_{i}$. On this approach, the speaker only has to know that there is a cover making the statement true (i.e., the speaker must only be sure of the existence of a derivation like the one in (31), where knowledge that veggies are in the baskets will point to the existence of such a derivation). The resulting analysis allows the full range of empirically attested interpretations, and says nothing about the way these various interpretations are derived and the factors that influence the derivation.

(33) The windows are open.

Sentences like (33) just mean that subparts of the given group of windows, in some packaging, are themes for (potentially plural) states of being open.

This semantics is very weak. The only information transmitted with the use of a cover-role is that there exists some derivation like the ones in (31, 32) - so, this incorrectly predicts that in a situation like BANK DOORS (8), repeated below, the speaker can say The doors are open on the collective interpretation (32a), allowing some doors to actually be closed, since a possible derivation exists as long as some doors are open.

(8) BANK DOORS, partially modified

a. Situation: The hearer works in a bank and must get to a safe that can only be reached via three consecutive doors.

b. Utterance: The doors are open.

c. Interpretation allowed in Theory IV: Some/All of the doors are open

d. Actual interpretation: All of the doors are open

This is much less information than would actually be transmitted by such an utterance in the situation in (8a). 
The meaning of plural definites

Similarly, this account incorrectly predicts that, in the situation from VEGGIES in (16a), the speaker can truthfully utter The vegetables are not too light for the big scale on the collective interpretation, even when the baskets are actually too light for the scale. After all, the possibility of putting all the baskets on the scale at once indicates that there is some way of making the sentence true.

As a result, by getting rid of the deictic cover variable, we have lost crucial information in these cases. While clearly too weak to stand on its own, this semantics is the perfect starting point for an account drawing on pragmatic factors to derive a stronger interpretation - after all, in any given scenario, hearers are able to figure out whether all or some windows/doors are required to be open, and which packaging is intended when baskets of vegetables need to be weighed.

\subsection{Desiderata for a proposal}

We can draw some interim conclusions from the discussion up to this point. In a theory of definite plurals, we must take into account that a deictic reference to packaging is necessary in some cases, e.g., VEGGIES (16) - without it, the semantics is too weak, as shown above. Note that in this case we are requiring deixis without speaker knowledge of the exact distribution of veggies into baskets; thus it would be deixis to intensionalised coverfunctions rather than covers (Kratzer forthcoming). At the same time, deixis to packaging is wrong in some cases, as in SAFE HOUSE and BANK DOORS (17) - because it makes non-maximality a matter of domain selection.

In addition to this flexibility in the reference to packaging, we still need to incorporate interlocutors' goals into the analysis.

In the proposal I lay out in the next section, I will introduce a deictic variable that will always include reference to hearer's goals, and will encode information about packaging only when such information is relevant to hearer's goals.

The pragmatic calculations will be particularised in the sense of Grice (1975b) (that is, they are derivable only in a specific context). Moreover, the calculation will be specific to each VP, reflecting the influence of the predicate on the distributivity and maximality of its arguments. 
Sophia A. Malamud

\section{A decision-theoretic proposal}

Following Parikh (1992), a number of researchers have used game and decision theory to model contextual factors in natural language interpretation. Here, I extend this line of research, and apply van Rooij's (2003) decision theory (DT) based definition of relevance to definite plurals. The proposal replaces the cover analysis, integrating Landman's (1996) recasting of Schwarzschild's proposal with a principled account of how and when the various interpretations arise. The chief innovation is therefore the unification of an underspecified semantics with a formal analysis of the influence of pragmatic factors on the truth-conditions of sentences with definite plurals. In this section, I first provide the formal concepts necessary for a decision-theoretic definition of relevance, and then propose an interpretation procedure for definite plurals in simple upward-entailing contexts in such situations as SAFE HOUSE and PAINTERS. Finally, I extend the account to downward-entailing contexts and complex sentences.

\subsection{Decision problems and relevance}

Agents in conversation are constantly modeling each other's minds. As a part of cooperative communication, each speaker aims to change the hearers' states of knowledge so as to help them progress towards their goals. This is the heart of the Cooperative Principle (Grice 1975b), and in particular, of the Relation (Relevance) maxim, as well as of other pragmatic theories such as Horn's (1984) R principle, and Sperber \& Wilson's (1986) Relevance Theory.

When interpreting an underdetermined utterance (in this case, one containing a definite plural), hearers select propositions which can influence their actions in achieving their goals. Agents' goals (and more) can be represented as decision problems (DeP) they are solving. A quantitative DeP is a triple $\langle(W, P), U, A\rangle$, where the probability function $P$ over the set of worlds $W$ represents the agent's beliefs, the utility function $U$ reflects the agent's preferences, and the set $A$ contains (mutually exclusive) actions the agent chooses from.

How can probabilities represent beliefs? One approach is to represent beliefs as subjective likelihoods that an agent assigns to states of affairs or worlds $w$, collected in the set of possible worlds, $W$. It is better to think of each world $w$ as representing, in fact, an equivalence class of possible worlds - worlds that are equivalent, for the purposes of the given situation. 
The meaning of plural definites

Let me expand on this briefly. In a recent treatment of quantity implicatures, Franke (2011) defines two worlds as being indistinguishable with respect to a set of alternatives ALT if every alternative in ALt is either true in both, or false in both. ${ }^{8}$ For the purposes of the relevance-based reasoning used here, the alternative actions $a \in A$ among which the hearer is choosing represent a generalisation over the alternative mental updates or states of the world represented by ALT. As a result, any two worlds in which the same action is optimal become one "state" or world for our purposes. This will have a profoundly simplifying effect on our relevance calculations.

A function $P: \wp(W) \mapsto[0,1]$ is a probability distribution on $W$ if it satisfies the following conditions: ${ }^{9}$

(34) Definition of a probability distribution $P$ on $W$

1. $P(W)=1$

2. for all $w \in W, P(w) \geq 0$

3. for any disjoint $p, q \subseteq W, P(p \cup q)=P(p)+P(q)$

(35) Max's possible beliefs in SAFE HOUSE: assuming the windows are $d_{1}$, $d_{2}$, and $d_{3}$.

\begin{tabular}{ll||ll} 
world & probability $(\mathrm{P})$ & world & $\mathrm{P}$ \\
\hline$w_{1}:$ all windows shut & $75 \%$ & $w_{5}:$ just $d_{1}$ and $d_{2}$ open & $3 \%$ \\
$w_{2}:$ just $d_{1}$ open & $5 \%$ & $w_{6}:$ just $d_{1}$ and $d_{3}$ open & $3 \%$ \\
$w_{3}:$ just $d_{2}$ open & $5 \%$ & $w_{7}:$ just $d_{2}$ and $d_{3}$ open & $3 \%$ \\
$w_{4}:$ just $d_{3}$ open & $5 \%$ & $w_{8}:$ all windows open & $1 \%$
\end{tabular}

In the example (35), note that the probabilities sum up to $100 \%$. The definition in (34) means that Max's belief in the proposition $d_{1}$ is open, for instance, is represented as $P\left(\left\{w_{2}, w_{5}, w_{6}, w_{8}\right\}\right)=P\left(w_{2}\right)+P\left(w_{5}\right)+P\left(w_{6}\right)+$ $P\left(w_{8}\right)=12 \%$.

Agents' beliefs might be changed if they learn new information. This can be modeled as reasoning with conditional probabilities. If $p$ and $q$ are propositions, the probability of $q$ given $p, P(q \mid p)$ is defined as in (36).

(36) Definition of conditional probability:

$$
P(q \mid p):=P(q \cap p) / P(p) \text {, provided } P(p) \neq 0
$$

$8 \mathrm{I}$ am grateful to an anonymous reviewer for detailed suggestions and discussion of the probabilities, worlds, and utilities that I use in this section and subsequently, and for pointing me to the very perspicuous discussion in Franke (2011).

9 I will abbreviate $P(\{w\})$ as $P(w)$ throughout. 
Sophia A. Malamud

For example, if Max learns the proposition $p$ that at least one of the windows is open, then his belief in the proposition $d_{1}$ is open changes as follows:

(37) (35, cont.) Max learns that at least one window is open:

$P\left(d_{1}\right.$ is open $\left.\mid p\right)=12 \% / P(p)=12 \% / 25 \%=48 \%$

Note, however, that the speaker usually does not have precise information about the hearer's prior beliefs/probabilities. Therefore neither the speaker in choosing an utterance, nor the hearer in calculating the correct interpretation, can rely on the speaker's knowledge of a probability distribution such as the one in (35). Instead, I will assume that both speakers and hearers base their calculations on a probability distribution that is as non-committal as possible, making the fewest assumptions possible about the hearer's beliefs. As I discuss below in $\S 4.1 .2$, this means that discourse participants assume a uniform distribution over the states of affairs, assigning equal probability to every world. Together with the one-world-per-action assumption, this means that speakers think of hearers as being in a state of perfect indecision between the actions in $A$, thinking each of them as equally likely to be optimal. ${ }^{10}$

Agents have preferences regarding the consequences of their actions, represented as numerical payoffs. Payoffs, when they are specified on an interval scale (i.e., a scale impervious to order-preserving linear transformations), ${ }^{11}$ are called utilities. Thus, a utility function maps action-world pairs to numbers: $U: A \times W \mapsto \mathbb{R}$.

The set of actions that the hearer is choosing from and the utility functions that determine the best choice of action can vary dramatically between different conversational contexts. Importantly, the speaker often does not have direct access to the actions and preferences of the hearer, and so must guess them. To illustrate, consider the following three examples. First, in SAFE HOUSE (2a-b), the speaker Mary is going on a road trip with the hearer Max, who has just asked the question Is the house going to be OK? The

10 Geurts \& Pouscoulous (2009) argue that beliefs about likely states of affairs don't impact the calculation of implicatures; Franke (2011) also uses flat probabilities that represent a lack of special assumptions about likelihood of different worlds.

11 That is, given any three outcomes of an action, $A, B$, and $C$, an agent can specify the rank order among them (e.g., $A>B>C$ ), as well as the ratio of the differences among the preferences (e.g., $(B-C) /(A-B)=2$ ). If the payoffs are interpreted as money, this means that an agent's choices are unchanged if the stakes are changed from pennies to thousands of dollars, or if, regardless of the outcome, the agent has to pay a certain fee. 
The meaning of plural definites

question becomes a QUD that the participants address (Roberts 1996). In this case, it is very clear which actions to include in the DeP - the basic dilemma is to go back to the house, or to continue with the trip. The utility function is also clear, and assigns the highest payoff to those actions that are consistent with the house being ok while minimising disruption to the trip: e.g. as in (38) (in my analysis, I will ultimately use a slightly different utility function for this situation). The speaker's estimates of the hearer's action set and utility function should be easy to make, since they are shared between the two conversational participants.

(35, cont.) Example of Max's preferences in SAFE HOUSE:

\begin{tabular}{lll} 
world & action & utility \\
\hline \hline \multirow{2}{*}{$w_{1}$ : all windows shut } & continue with the trip & 5 \\
& go back to the house & o \\
\hline \multirow{2}{*}{ all other worlds } & continue with the trip & 1 \\
& go back to the house & 3
\end{tabular}

Second, consider a reader of a novel, enjoying her book. In this case, the action set facing the reader of a specific sentence (utterance) needs some discussion. I will follow Roberts (1996), among others, and assume that assertions address Questions Under Discussion, which can be implicit. In the case of a novel, the set of alternative actions consists simply of the different ways in which the current (inferred) QUD can be addressed. The utilities are higher for propositions that resolve the QUD most completely - and for fiction it is also natural to assume higher utilities for more interesting, stylistically pleasing, or perhaps thought-provoking, propositions resolving the QUD. In the context of a novel, the immediate QUD is often inferred from the proposition resolving it, rather than raised explicitly (see, e.g., Hulstijn 1997, van Kuppevelt 1995 for a discussion of how QUDs might be raised) - in which case we should perhaps assign higher utilities to propositions that imply (and then resolve) more interesting and aesthetically pleasing QUDs. ${ }^{12}$ It seems that the speaker (writer) can estimate the action set more easily than a utility function tied to literary taste. However, assuming a sympathetic reader and a talented writer, we would not be wrong to assume that the speaker's estimate is close to the hearer's reality.

12 Robin Clark (p.c.) points out that English has a narrative the that never seems to require maximality/uniqueness.

(i) I knocked on John's door; someone was behind the window, but no one answered the door. 
Finally, consider a novice extra-terrestrial geology scholar hearing or reading a report about Martian geology. As in the case of the novel, the relevant actions are simply the different mental updates that the hearer makes in response to an utterance in the report. The utility function is completely different, however: now, the more information the hearer receives, the higher the payoff. That is, the QUD in this case is, What is the actual world like? As I discuss in $\$ 4.1 .2$ below, utility in such cases reduces to informativity/strength.

Recent experimental work suggests that conversational participants will prefer above all to resolve the current QUD - that is, to find at least one complete answer for the QUD (Zondervan, Meroni \& Gualmini 2008). ${ }^{13}$ In the terminology of decision theory, the desire to resolve the QUD can be expressed as the desire to get rid of the state of indecision (van Rooij 2003): the agent prefers above all to resolve the decision problem. Formally, this is defined as follows.

(39) a. An action a dominates all other actions when in each world no action has a higher utility than $a$.

b. A decision problem is resolved when one of the actions in $A$ dominates all other actions.

Based on (39), we can define what it means for a proposition to resolve a decision problem, as follows.

(40) a. A decision problem updated with a proposition $q, \operatorname{DeP}[q]$ is one in which the probabilities $P(w)$ in the DeP have been changed to probabilities conditional on $q, P(w \mid q)$.

b. A proposition $q$ can be said to resolve a decision problem DeP if DeP is not resolved, while $\operatorname{DeP}[q]$ is resolved.

Note that based on the utilities and beliefs represented in $(35,38)$ for the situation in SAFE HOUSE, no single action dominates, so Max's decision problem is unresolved.

(ii) A man walked into a bar and the bartender asked him ...

There is no implication that John's house has only one window, or the bar only one bartender; new ones can be introduced as they become relevant to the narrative. This demonstrates especially clearly the strategic thinking involved in constructing a narrative: the unmentioned windows/bartenders are simply irrelevant. As in the plural DuNEs (4) example, their mention will not help the speaker address the QUD.

13 The participants will need to choose the current QUD among the many possible and unresolved ones - while very important, the computation behind this choice lies outside the scope of this paper (but see Roberts 1996 for one view). 
The meaning of plural definites

The action set $A$ corresponds to a QUD, because each action $a$ gives rise to a proposition $p_{a}$ - specifically, to the set of worlds in which that action dominates. (With our simplifying assumption that an action is optimal in exactly one world, this means that each $p_{a}$ is a singleton.) As a result, $A$ gives rise to a set of propositions $p_{a}$, that is, to a question $Q_{A}$, Which action is an optimal one?. ${ }^{14}$ In SAFE HOUSE, this question is Should we go back to the house or not? and, based on the utilities in (38), it is $Q_{A}=$ $\left\{\left\{w_{1}\right\},\left\{w_{2}, w_{3}, w_{4}, w_{5}, w_{6}, w_{7}, w_{8}\right\}\right\}$, a partition.

Thus, in contexts in which no actual question has been asked, the hearer's decision problem corresponds to a QUD.

Tying together actions, utilities, and propositions is the notion of expected utility. The expected utility of an action is essentially the average of the payoffs associated with that action in different scenarios:

(41) Expected Utility of an action $a$ :

$$
E U(a)=\sum_{w \in W} P(w) \times U(a, w)
$$

(42) (35, cont.) Example of expected utilities of actions in SAFE HOUSE:

a. $E U$ (continue with the trip) $=.75 \times 5+.25=4=$ $P($ all windows closed $) \times 5+P($ at least one window open $) \times 1$

b. $E U($ go back to the house $)=.25 \times 3=.75=$ $P($ all windows closed $) \times 0+P($ at least one window open $) \times 3$

Rational agents seek to maximise their expected utilities. Therefore, Max will choose the action that has the highest expected utility: in SAFE HOUSE (42), to continue the trip.

When agents learn new information, and thus change the probabilities they assign to the different worlds, their choices may change. The expected utility of an action $a$ after learning a proposition $q$ is defined as follows:

(43) Expected Utility of an action $a$ after learning $q$ :

$$
E U(a \mid q)=\sum_{w \in W} P(\{w\} \mid q) \times U(a, w)
$$

14 In the semantics of Groenendijk \& Stokhof (1984), not every set of propositions is a good denotation for a question - only those that form partitions are. That is, the propositions within the set have to be disjoint. However, as argued, for instance, in van Rooij (2003), some questions actually do not correspond to partitions. For the purposes of this paper, my simplifying assumptions make $Q_{A}$ a partition. However, in general, the propositions in the $Q_{A}$ may not be disjoint, so I am calling the set of propositions $Q_{A}$ a question in van Rooij's (2003) sense. 
Based on this, we can determine the maximum expected payoff for an agent facing a decision problem: (44a) defines the value of making a decision with current knowledge, while (44b) gives the value of making a decision after learning the proposition $q$.

(44) a. $U V($ choose now $)=\max _{a \in A} E U(a)$

b. $\quad U V($ learn $q$, then choose $)=\max _{a \in A} E U(a \mid q)$

Max's $U V$ (choose now) in SAFE HOUSE $(35,38)$ is 4 , as calculated in (42). His $U V$ (learn at least one window is open, then choose) is derived below.

(45) (35, cont.) Max's expected payoff in SAFE HOUSE after learning $p=$ at least one window is open:

a. $E U($ continue with the trip $\mid p)=P($ all windows closed $\mid p) \times U\left(\right.$ continue,$\left.w_{1}\right)$ $+P($ at least one window open $\mid p) \times U\left(\right.$ continue, $w_{2}$ or $w_{3} \ldots$ or $\left.w_{8}\right)=0 \times 5+1 \times 1=1$

b. $E U($ go back home $\mid p)=P($ all windows closed $\mid p) \times U\left(\right.$ go back, $\left.w_{1}\right)$ $+P($ at least one window open $\mid p) \times U\left(\right.$ go back, $w_{2}$ or $w_{3} \ldots$ or $\left.w_{8}\right)=$ $0 \times 0+1 \times 3=3$

c. $U V($ learn $p$, then choose $)=3$

The difference between the two values given in (44) is exactly the difference that the proposition $q$ makes to the decision-maker, the proposition's utility (46). This notion was used by Parikh (1992, 2000) and van Rooij (2003), among others, in analyses of conversational implicature, question and answer interpretation, and other interpretational phenomena.

(46) $U V(q)=U V($ learn $q$, then choose $)-U V$ (choose now)

As van Rooij (2003) notes, this definition gives rise to a relevance scale: proposition $p$ is more relevant, or better to learn, than $q$ with respect to a decision problem (DeP) if its utility is higher.

(47) $p>_{D e P} q$ iff $U V(p)>U V(q)$.

In the SAFE HOUSE example, with utilities as in (38), the value of learning $p=$ at least one of the windows is open is negative, and identical to the value of learning $q=$ all of the windows are open (48), making these two propositions equally relevant. 
The meaning of plural definites

(48) (35, cont.) Max's value in SAFE HOUSE for $p=$ at least one window is open

vs. $q=$ all of the windows are open:

a. $U V($ learn $q$, then choose $)=E U($ go back to the house $\mid q)=$ $P$ (all windows closed $\mid q) \times U\left(\right.$ go back to the house, $\left.w_{1}\right)$

$+P($ at least one window open $\mid q) \times U$ (go back, in other worlds)

$=0 \times 0+1 \times 3=3$

b. $U V(p)=U V(q)=U V($ learn, then choose $)-U V($ choose now $)$

$=3-4=-1$

The specific utility function may vary from context to context - it will be different depending on whether the hearer is interested in reading a report on Martian geology or resolving a simple dilemma (e.g., in SAFE HOUSE should I go back to the house or go on with the trip?). For the core examples in SAFE HOUSE, PAINTERS, BANK DOORS etc., we can use the idea that conversational participants prefer above all to resolve the current QUD which corresponds to the DeP. That means that the utility function assigns payoffs that depend only on the probabilities of the propositions $p_{a} \in Q_{A}$. This can be modelled by the utility function $U_{01}$, defined in (49).

(49) Utility function to be used for all calculations below:

$$
U_{01}(a, w)=\left\{\begin{array}{l}
1 \text { if } a \text { is optimal in } w \\
0 \text { if } a \text { is not optimal in } w
\end{array}\right.
$$

Together with the assumption that each world in fact represents an equivalence set of worlds in which the same action is optimal, this results in the "diagonal" utilities, where exactly one action is optimal in each world, and exactly one world has a particular action as optimal. ${ }^{15}$

In this case, the overall expected utility of each action $a$ becomes just the probability that the hearer is in a world in which that action is optimal - the probability of the proposition $p_{a}$. I will assume the utility function $U_{01}$ for the central examples analysed in this section.

Recall that a proposition resolves a decision problem if, after it is learned, at least one action dominates $(39,40)$. Together with the simplifying assumption that, in a world, exactly one action is optimal, this means that the

15 I am grateful to an anonymous reviewer for suggesting diagonal utilities, and pointing out that decision problems using diagonal utilities and flat prior probabilities have aready been used in formal pragmatics, e.g. Franke (2011). 
decision problem will give rise to a partition $Q_{A}$, whose propositions do not overlap. A proposition can resolve a decision problem either by changing the hearer's probability of different answers, or by eliminating some of the answers entirely. We will concentrate on the latter possibility. ${ }^{16}$ Defining the utility of propositions and their resulting ordering (47) becomes simple in this case, if we pursue the idea that the agent's goal is to resolve the DeP (50). The definition in (50) essentially rewards those propositions that eliminate more cells of the partition $Q_{A}$.

(50) Proposition $p$ is more relevant (better to learn) for resolving DeP than $q\left(p>_{D e P} q\right)$ iff $p$ eliminates more actions as non-optimal than $q$ does

It is easy to see that for the special case of diagonal utilities in which there is a one-to-one correspondence between optimal actions and worlds, the two definitions (47) and (50) will yield the same ordering for propositions. For example, in SAFE HOUSE, the proposition $p=$ at least one window is open eliminates the action of continuing with the trip, and therefore resolves the DeP. So does the proposition $q=$ all windows are open, making the two propositions equally relevant (cf. 48). The ordering in (50) ignores the numerical utility values for propositions, and simply looks at what actions are optimal; as a result, the utility function $U_{01}$ is sufficient.

Van Rooij (2003), among others, proposes a second clause for the definition of relevance ordering (50): $p>_{D e P} q$ if $p$ eliminates the same number of actions as $q$, but $q$ entails $p$ (i.e., $q$ is over-informative). However, this is not needed in my proposal for definite plurals, as I will demonstrate below.

When more than one action per world is optimal, it becomes harder to define a measure like (50) of how much closer a proposition brings the hearer to resolving the DeP - if two actions are both optimal, eliminating one of them should not be rewarded. ${ }^{17}$ In this case, propositions with higher utility are those that allow the hearer to come closer to an answer for the

16 That is, given the assumption that the hearer's prior beliefs are estimated to be a flat probability distribution, when a proposition is learned, all cells of the QUD consistent with it have equal probability. I thank an anonymous reviewer for pointing out that a standard Bayesian update together with flat priors yields this result. When the QUD is $W$, What is the world like?, the probabilities will mimic the newly-learned proposition (Potts 2006). A probability distribution $P$ mimics the proposition $q \subseteq W$ iff:

(i) for all $w \notin q, P(\{w\})=0$

(ii) for all $w_{i}, w_{j} \in q, P\left(\left\{w_{i}\right\}\right)=P\left(\left\{w_{j}\right\}\right)$

17 I am grateful to an anonymous reviewer for bringing up this point 
The meaning of plural definites

mention-some question, Which actions are optimal in the actual world? ${ }^{18}$

In making an utterance, speakers aim to resolve the hearers' DeP, or, rather, their estimate of the hearers' DeP. ${ }^{19}$ The estimate will be good enough if speakers have an idea about the actions their hearers are choosing from, and if they can figure out the effect of learning various propositions on that action set. As a result, in a situation in which the speaker's utterance can have several different interpretations, all of which point to the same action as optimal, this underspecified utterance will resolve the underlying DeP perfectly, and thus will be acceptable to both the speaker and the hearer.

\subsection{The basic proposal}

A relevance ordering such as the one in (50) allows the hearer to choose an appropriate interpretation for the underspecified definite, and is built into the definition of the variable REL (51). ${ }^{20}$

(51) The relevance operator (first version)

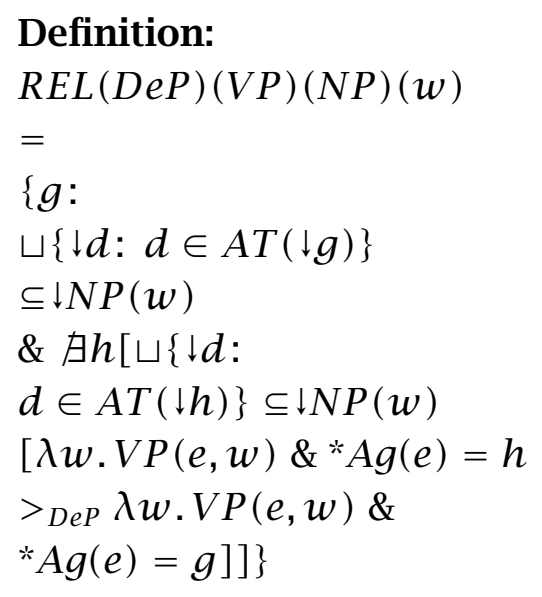

\section{Paraphrase:}

REL takes the decision problem, VP and NP denotations, and a world, and outputs a set of individuals $g$ (groups or sums). When you boil $g$ down to singularities, those individuals are atoms in the NP denotation (e.g., the boys). No other such NP-made (e.g., boy-made) thing $h$ is more relevant than $g$ - that is, there is no $h$ such that it's better to learn that " $h$ VPs" than that " $g$ VPs" with respect to the given decision problem.

18 See the example in (11), and discussion preceding it, for an illustration of a mention-some question.

19 While practically all linguists agree that conversational participants represent each other's goals and knowledge during a conversation, the processes and representations utilised during such modelling are the subject of much ongoing research, and lie outside the scope of this paper.

20 REL stands for "relevant", instead of a similar variable Op for "optimal” proposed by van Rooij. If $x$ is a pure atom, $\downarrow x=x$ (Landman 1989). If $x$ a sum, rather than an atom, I assume $\downarrow x=x$. 
The operator containing REL introduces a set of alternatives $-O_{\text {Rel }}$ is a function from VP and NP denotations to the set of optimally relevant propositions (52).

$$
O_{R e l}=\lambda w \cdot \lambda P . \lambda q \cdot \lambda x \cdot \lambda e \cdot x \in R E L(D e P)(P)(q)(w) \& P(e, w) \& * A g(e, x)
$$

The underspecified and flexible semantics assigning a cover-role to the NP is disambiguated by the pragmatic operator (hence the use of the plural-roles, rather than cover-roles for the subparts $g$ and $h$ of the NP). REL replaces Schwarzschild's (1991) $\operatorname{Cov}_{i}$ in encoding the underspecification and contextdependence. The REL operator comes as part of the VP, following syntactic assumptions in Schwarzschild (1991) and other literature on distributivity (53 presents the structure).

(53)

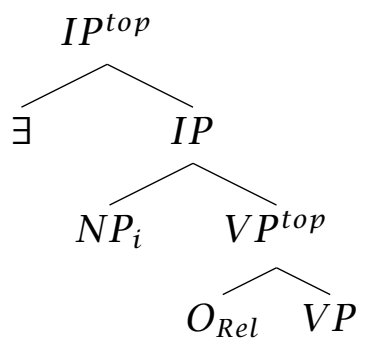

The alternative interpretations are built up compositionally, and a set of optimally relevant ones is selected by REL. The sentential existential quantifier converts this set into a single proposition at the top. This last operation is the one introduced for the Hamblin semantics in Kratzer \& Shimoyama (2002). The derivation schema for the entire sentence, following the tree in (53), is given in (54). While I build the relevance operator into the object language, I do not believe that such an object-language operator is necessary. Its position immediately below the existential allows us to interpret the corresponding calculations as occurring automatically in every sentence that contains underspecified definite plurals. I will continue to use the object-language $O_{\text {Rel }}$ for perspicuity and to uphold the parallel with Schwarzschild's $\operatorname{Cov}_{i}$. I will briefly discuss this issue further in $\$ 5$.

$$
\begin{array}{r}
\llbracket V P^{t o p} \rrbracket=\llbracket\left[O_{R e l} V P\right] \rrbracket=\lambda w \cdot \lambda q \cdot \lambda x \cdot \lambda e \cdot x \in R E L(D e P)(V P)(q)(w) \& \\
V P(e, w) \& * A g(e, x) \\
\llbracket I P \rrbracket=\llbracket\left[N P_{i} V P^{t o p}\right] \rrbracket=\lambda w \cdot \lambda x \cdot \lambda e \cdot x \in R E L(D e P)(V P)(N P)(w) \& \\
V P(e, w) \& * A g(e, x) \\
\llbracket I P^{t o p} \rrbracket=\llbracket[\exists I P] \rrbracket=\lambda w \cdot \exists p[p \in\{\lambda v \cdot[g \in R E L(D e P)(V P)(N P)(v) \& \\
V P(e, v) \& * A g(e)=g]: g \in R E L(D e P)(V P)(N P)(w)\} \& p(w)]
\end{array}
$$


The meaning of plural definites

These tools allow us to calculate the correct levels of maximality and distributivity for underspecified definite plurals. Here are a few worked-out examples to illustrate the schema in (54). First, to address (non)maximality, consider PAINTERS (7), analysed below (55).

(55) a. PAINTERS decision problem: Hearer is preparing his house for the arrival of painters, who will paint all the window-frames. He has to decide if he can relax till they arrive (Action 1), or if he still needs to do something to prepare for the painters (Action 2).

Consider an agent facing the problem in (55a). If he hears (55b), he has to select at least one of the interpretations from the pool of propositions (55c) as the optimal reading of the underspecified literal statement.

(55) b. Utterance: The windows are open.

$\lambda w . \exists p[p \in\{g(w) \in R E L(D e P)($ win $)($ open $)(w) \& g(w)$ is open $\} \& p]$

c. Pool of propositions REL chooses from:

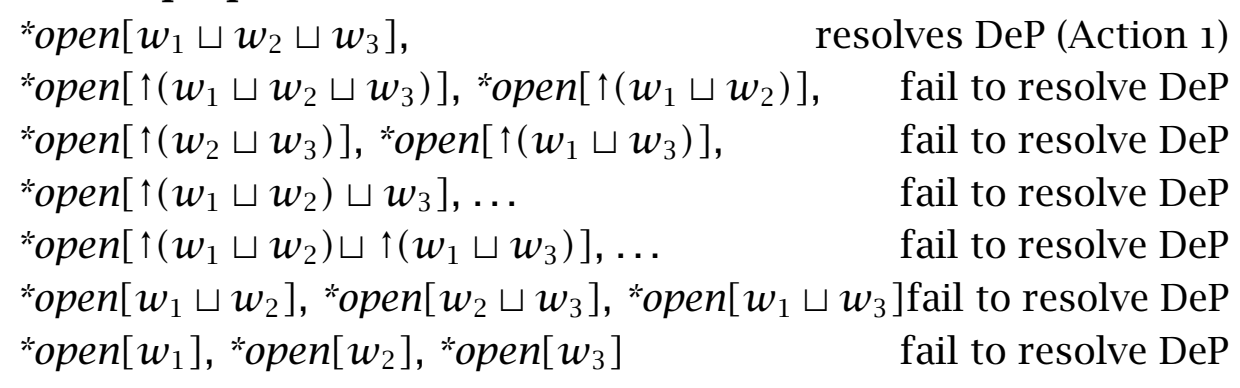

The propositions themselves are built up compositionally all at once, following the schema in (54). While the proposition first listed resolves the decision problem (by pointing to Action 1), all others fail to do so, since they are compatible both with a scenario in which all windows are closed, and one in which some are open and some closed. So the first, maximal, proposition is the only relevant interpretation, as reflected in $(55 \mathrm{~d})$.
d. The derived interpretation: (all of the windows are open) $\exists p\left[p \in\left\{\exists e . * \operatorname{open}(e) \& w_{1} \sqcup w_{2} \sqcup w_{3} \sqcup w_{4} \sqcup w_{5}=* A g(e)\right\} \& p\right]$

A built-in consequence of the framework is this: the only way to force a maximal interpretation is for REL to produce a single most relevant proposition, so that the existential quantification over propositions is over a singleton set. In addition, we inherit from Landman the fact that maximal interpretations are simply fully distributive ones, in which a unique optimal proposition 
involves distributive predication over a sum. To illustrate what happens when these conditions fail to hold, consider the example SAFE HOUSE (2), analysed in (56) below.

(56) a. SAFE HOUSE decision problem: Before a thunderstorm, the hearer has to decide whether to go on with daily business (Action 1 ) if all windows are closed or return home (Action 2) if some windows are open.

In SAFE HOUSE, an agent is facing the problem in (56a). If he hears the same utterance as before $(55 \mathrm{~b}, 56 \mathrm{~b})$, then all of the propositions in the pool resolve the decision problem (by pointing to Action 2), and therefore all are optimally relevant.

(56) b. Utterance - same as in (55b): The windows are open.

$\lambda w . \exists p[p \in\{g(w) \in R E L(D e P)($ win $)($ open $)(w) \& g(w)$ is open $\} \& p]$

c. Pool of propositions REL chooses from:

*open $\left[w 1 \sqcup w_{2} \sqcup w_{3}\right], \quad$ resolves DeP (Action 2)

*open $\left[\uparrow\left(w_{1} \sqcup w_{2} \sqcup w_{3}\right)\right]$, *open[ $\left.\uparrow\left(w_{1} \sqcup w_{2}\right)\right]$, resolves DeP (Action 2)

*open $\left[\uparrow\left(w_{2} \sqcup w_{3}\right)\right]$, *open[ $\left.\uparrow\left(w_{1} \sqcup w_{3}\right)\right], \quad$ resolves DeP (Action 2)

*open $\left[\uparrow\left(w_{1} \sqcup w_{2}\right) \sqcup w_{3}\right], \ldots \quad$ resolves DeP (Action 2)

*open $\left[\uparrow\left(w_{1} \sqcup w_{2}\right) \sqcup \uparrow\left(w_{1} \sqcup w_{3}\right)\right], \ldots \quad$ resolves DeP (Action 2)

*open $\left[w_{1} \sqcup w_{2}\right]$, *open $\left[w_{2} \sqcup w_{3}\right], \ldots \quad$ resolves DeP (Action 2)

*open $\left[w_{1}\right]$, *open $\left[w_{2}\right]$, *open $\left[w_{3}\right] \quad$ resolves DeP (Action 2)

This means that, whenever REL fails to winnow the set down to a single fully distributive proposition, the existential quantification over propositions will result in a weaker meaning, which states that one of the most helpful propositions is true. If the hearer doesn't care which windows are open, all propositions stating that at least one of the windows is open are equally most helpful. Consequently, the existential quantification over propositions creates the effect of existential quantification over the windows (i.e., the non-maximal reading (56d)).

(56) d. The derived interpretation: (some of the windows are open) $\exists p\left[p \in\left\{\exists e . * \operatorname{open}(e) \& w_{1}={ }^{*} A g(e), \exists e .{ }^{*} \operatorname{open}(e) \& w_{2}={ }^{*} A g(e), \ldots\right.\right.$ $\left.\left.\ldots \exists e .{ }^{*} \operatorname{open}(e) \& w_{1} \sqcup w_{2} \sqcup w_{3}=* A g(e)\right\} \& p\right]$

The theory of interpretation proposed here, thus, derives competing interpretations compositionally; the resulting reading is essentially a disjunction of all the optimally relevant alternatives. This means that, for those exam- 
The meaning of plural definites

ples in which a salient "packaging" forces a particular domain selection, all surviving alternatives will conform to this packaging. In contrast, when it is irrelevant which specific singularities are chosen (as in SAFE HOUSE), a variety of covers will survive, creating a reading that is indeterminate between them. ${ }^{21}$

With the basics of the proposal laid out, we can now consider more complex sentences, extending the analysis to downward-entailing contexts and coordinated predicates.

\subsection{Further applications}

\subsubsection{Downward-entailing contexts}

Returning to the data in the first section, we must account for the tendency of maximality judgments to switch under negation, and in other downwardentailing contexts. ${ }^{22}$

(57) a. I doubt that Peter saw the linguists. (I doubt he saw any)

b. I believe that Peter saw the linguists. (I believe he saw all of them)

In selecting the most relevant children or linguists, the hearers must consider the entire linguistic context (including negation or doubt), and not just the basic clause composed of the VP and NP. This means that the Decision-Theoretic relevance operator in (51) must be revised slightly, so that the propositions in the pool are based on the entire utterance, and allowing non-agent NPs, such as the object in (57), to be resolved.

In order to "re-constitute" the entire sentence while keeping track of the predicate-argument pair whose indeterminacy is being resolved, we use indexing: the predicates (verbs, VPs, or adjectives) are numbered, and the theta-role of the argument whose subparts are in question is indicated. ${ }^{23} \mathrm{~A}$ sentence containing an underspecified predicate-argument pair is co-indexed

21 I thank an anonymous reviewer for emphasising the importance of this model.

22 This is, indeed, just a tendency, since judgments do not switch in SAFE HOUSE or PAINTERS:

(i) The windows are open.

(SAFE HOUSE: some of them; PAINTERS: all of them)

(ii) The windows are not open. (SAFE HOUSE: not any of them; PAINTERS: not all of them)

23 I remain neutral on the important syntactic question of whether adjectives can assign syntactic theta-roles. Instead, I use the term to denote semantic roles that semantic arguments play in eventualities (both states and events). 
with such a pair: I use $n$ as a variable over numbers, and $\theta$ as a variable over theta-roles; this means that $X_{n \theta_{n}}$ is a syntactic object $X$ (usually, an IP) which contains a predicate with the index $n$ and an NP to which that predicate assigns the theta-role $\theta_{n}$ (for perspicuity, I will index the NP with the theta-role assigned to it, $N P_{\theta_{n}}$. As a result, the interpretation $\llbracket X_{n \theta_{n}} \rrbracket$ of such a syntactic object contains $\llbracket$ Predicate $\left._{n} \rrbracket(e, w) \&^{c} \theta_{n}(e)=\llbracket N P_{\theta_{n}} \rrbracket\right)$.

In constructing the pool of potentially relevant propositions, we substitute various combinations of the subparts from the denotation of the $N P_{\theta_{n}}(h$ and $g$ in (58)) into that NP's slot in $\llbracket X_{n \theta_{n}} \rrbracket$. I use the notation $\llbracket X \rrbracket[h / \llbracket N P \rrbracket]$ to indicate the denotation of $\mathrm{X}$ in which the object $h$ substitutes for the denotation of the NP.

(58) The relevance operator (second version)

$$
\begin{aligned}
& \text { Definition: } \\
& \text { Paraphrase: } \\
& R E L(D e P)\left(X_{n \theta_{n}}\right)(w) \\
& = \\
& \{g: \\
& \sqcup\{\downarrow d: d \in A T(\downarrow g)\} \\
& \subseteq \downarrow \llbracket N P_{\theta_{n}} \rrbracket(w) \\
& \& \neg \exists h \\
& {[\sqcup\{\downarrow d: d \in A T(\downarrow h)\}} \\
& \subseteq \downarrow \llbracket N P_{\theta_{n}} \rrbracket(w) \\
& {\left[\lambda w . \llbracket X_{n \theta_{n}} \rrbracket\left[h / \llbracket N P_{\theta_{n}} \rrbracket\right]\right.} \\
& >_{D e P} \\
& \text { REL takes a DeP, an X containing a predicate }{ }_{n} \\
& \text { and its argument } \mathrm{NP}_{\theta_{n}} \text {, and a world } \\
& \text { and outputs a set of groups or sums } g \text {. } \\
& \text { The singularities underlying } g \text { are } \\
& \text { atoms in the } \mathrm{NP}_{\theta_{n}} \text { denotation (e.g., the doors). } \\
& \text { No other such } \mathrm{NP}_{\theta_{n}} \text {-made (e.g., door-made) } \\
& \text { thing } h \text { is more relevant than } g \text {-i.e., there } \\
& \text { is no } h \text { such that learning the proposition } \\
& \llbracket X \rrbracket \text { about } h \text { fulfilling the predicate }{ }_{n} \\
& \left.\left.\left.\lambda w . \llbracket X_{n \theta_{n}} \rrbracket\left[g / \llbracket N P_{\theta_{n}} \rrbracket\right]\right]\right]\right\} \quad \text { learning } \llbracket X \rrbracket \text { about } g \text { fulfilling the predicate }{ }_{n} \text {. } \\
& =\text { Spelling out the propositions whose relevance is being compared: } \\
& \left\{g: \sqcup\{\downarrow d: d \in A T(\downarrow g)\} \subseteq \downarrow \llbracket N P_{\theta_{n}} \rrbracket(w)\right. \\
& \& \neg \exists h\left[\sqcup\{\downarrow d: d \in A T(\downarrow h)\} \subseteq \downarrow \llbracket N P_{\theta_{n}} \rrbracket(w)\right. \\
& \&\left[\lambda w .\left[\ldots \llbracket \text { Predicate }_{n} \rrbracket(e, w) \& * \theta_{n}(e)=h \ldots\right]>_{D e P}\right. \\
& \left.\left.\left.>_{D e P} \lambda w .\left[\ldots \llbracket \text { Predicate }_{n} \rrbracket(e, w) \& * \theta_{n}(e)=g \ldots\right]\right]\right]\right\}
\end{aligned}
$$

This modification can derive contextually appropriate interpretations of examples such as (57), using a procedure that completely mirrors the derivation of the examples in the previous section. 
The meaning of plural definites

\subsubsection{Structures with predicate coordination}

Now that the expressions considered by REL are entire utterances, a technical question arises in predicate coordination, especially when the levels of maximality or distributivity of the same NP are different for the two predicates, as in (59), an example I will name EMPTY HOUSE: how are the propositions for REL constructed? And how are the results of the relevance calculation combined to yield the reading that is optimally relevant for both predicates?

\section{(59) Coordinated predicates, EMPTY HOUSE}

a. Situation: The owner of a house has not occupied or seen it for a year, and is expecting some guests. To be ready for the arrival of the guests, the house must be in good repair (no windows broken), and the rooms must be aired (by opening some windows). The speaker is a friend of the owner, who goes to check on the situation, and to determine which of the four actions must be taken: $a_{1}=$ repair broken windows and open some, $a_{2}=$ open some windows (no repairs needed), $a_{3}=$ repair broken windows (some are already open), $a_{4}=$ do nothing (no repairs needed, and the place is being aired).

b. The windows are open and unbroken.

c. The windows are closed but broken.

Since the NP-parts involved in the two predicates may be different, the most relevant such subparts must be calculated separately for the two predicates. In constructing the propositions from which the REL chooses optimal ones for each predicate, the other predicate will remain underspecified. The REL defined in (58) produces a separate calculation for each predicate-NP pair, yielding for each predicate its own set of optimal subparts of the NP. The pool of propositions for each calculation is built by cycling through different subparts of the NP with one predicate, then the other, as in the calculation (62) below for (59b).

Once the sets of optimal NP-subparts are chosen by REL, they must be reincorporated into the interpretation of the whole utterance. The operator $O_{R e l}$ (52) is changed to achieve this, taking the sets of optimal subparts of the NP, and combining these pointwise with the correct predicates to yield the final set of propositions. The top existential quantifier selects a proposition from this set, as before. Since $O_{\text {Rel }}$ triggers a relevance calculation which considers 
the whole utterance, it must be introduced at the top of the sentence. Since the REL-sets of optimally relevant subparts are indexed to the theta-role of the corresponding predicate, they are matched to that theta-role in the reintegrated propositions. In this structure, the underspecified NP-predicate pairs become visible to $O_{R e l}$ through co-indexing. The new structure is shown in (6o) below.

(6o)

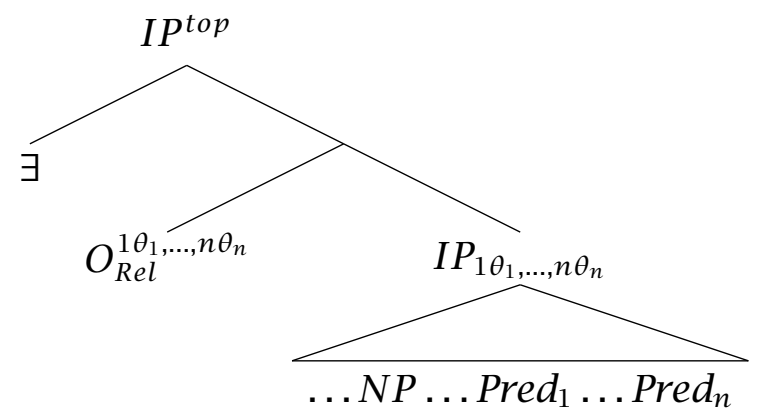

$O_{R e l}$ takes a proposition containing underspecified NP and predicate denotations, and builds a set of propositions that are simultaneously optimal with respect to each NP-predicate pair. It does so by plugging in NP-parts from the REL-set of each predicate into the total proposition where that predicate's theta-role is assigned (61).

$O_{R e l}^{1 \theta_{1}, \ldots, n \theta_{n}}=\lambda w \cdot \lambda \phi . \lambda x_{1} \ldots \lambda x_{n} . \lambda e_{1} \ldots \lambda e_{n} . \phi=\llbracket X_{1 \theta_{1} \ldots n \theta_{n}} \rrbracket \&$ $x_{1} \in R E L(D e P)\left(X_{1 \theta_{1}}\right)(w) \& \ldots x_{n} \in R E L(D e P)\left(X_{n \theta_{n}}\right)(w) \&$ $\llbracket X \rrbracket\left[x_{1} / \llbracket N P_{\theta_{1}} \rrbracket \ldots x_{n} / \llbracket N P_{\theta_{n}} \rrbracket\right]=$ spelling out the contents of the combined proposition $=\lambda w \cdot \lambda \phi \cdot \lambda x_{1} \ldots \lambda x_{n} . \lambda e_{1} \ldots \lambda e_{n} . \phi=\llbracket X_{1 \theta_{1} \ldots n \theta_{n}} \rrbracket \&$ $x_{1} \in R E L(D e P)\left(X_{1 \theta_{1}}\right)(w) \ldots x_{n} \in R E L(D e P)\left(X_{n \theta_{n}}\right)(w) \&$ $\&\left[\ldots \llbracket\right.$ Pred $\left._{1} \rrbracket\left(e_{1}, w\right) \& * \theta\left(e_{1}\right)=x_{1} \ldots \llbracket \operatorname{Pred}_{n} \rrbracket\left(e_{n}, w\right) \& * \theta\left(e_{n}\right)=x_{n}\right]$ 
The meaning of plural definites

Consider now EMPTY HOUSE (59a, b), whose structure is given in (62a).

(62) Analysis for (59b): The windows are open and unbroken.

a.
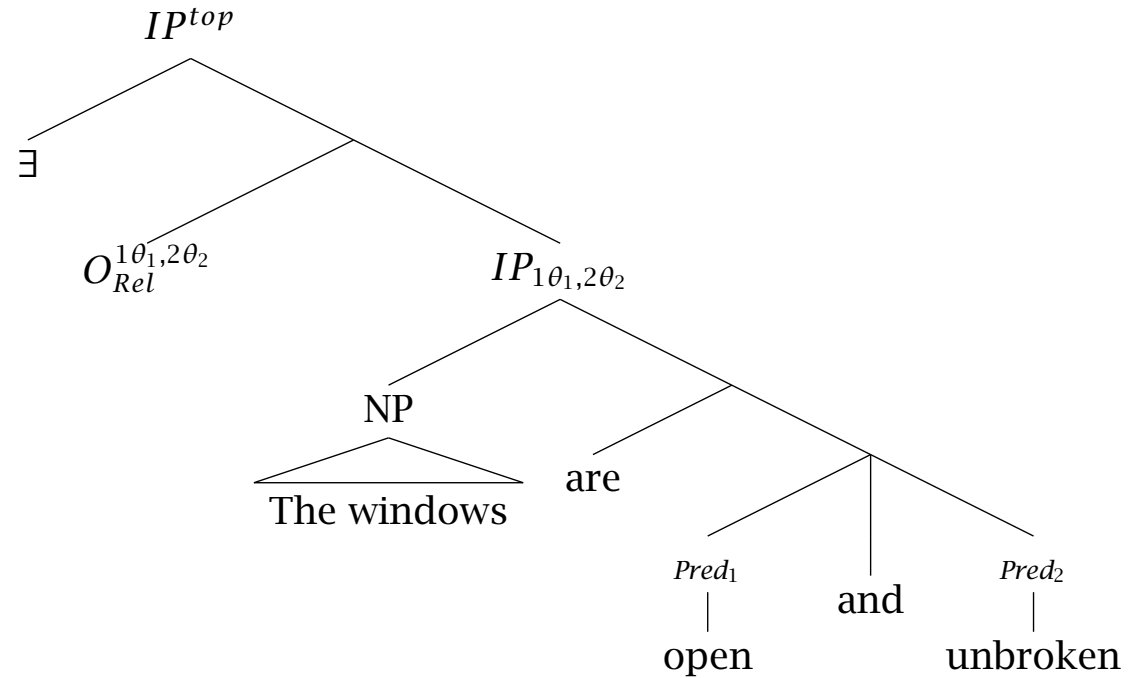

The lower IP is an underspecified proposition with two predicate-NP pairs: the windows are open, and the windows are unbroken (62b). These pairs are coindexed with $O_{\text {Rel }}$, triggering the calculation of two different sets of windows - those optimally relevant for open (62c), and those relevant for unbroken (62d).

$$
\begin{aligned}
& \text { b. } \llbracket I P_{1 \theta_{1}, 2 \theta_{2}} \rrbracket=\lambda w .{ }^{*} \operatorname{open}\left(e_{1}, w\right) \&{ }^{c} \theta_{1}\left(e_{1}\right)=\uparrow \sigma x^{*} \text { windw }(x, w) \& \\
& { }^{*} \text { unbroken }\left(e_{2}, w\right) \&{ }^{c} \theta_{2}\left(e_{2}\right)=\uparrow \sigma x^{*} \text { windw }(x, w)
\end{aligned}
$$

In this situation, as long as some windows are open, the actions which include window-opening are eliminated (62c); I use only two windows, $d_{1}$ and $d_{2}$.

c. Pool of propositions for Pred $_{1}$ :

*open $\left(d_{1}\right) \&$ *unbr $(\uparrow \sigma x *$ window $(x, w)) \quad$ eliminates $a_{1}$ and $a_{2}$

*open $\left(d_{2}\right) \&$ *unbr $(\uparrow \sigma x *$ window $(x, w)) \quad$ eliminates $a_{1}$ and $a_{2}$

*open $\left(d_{1} \sqcup d_{2}\right)$ \& *unbr $(\uparrow \sigma x *$ window $(x, w)) \quad$ eliminates $a_{1}$ and $a_{2}$ Thus, $\operatorname{Rel}(\operatorname{DeP})\left(I P_{1 \theta_{1}}\right)(w)=\left\{d_{1}, d_{2}, d_{1} \sqcup d_{2}\right\}$

It is clear that any proposition stating that a window is open is equally relevant, resulting in several alternative propositions corresponding to different subsets of windows (62e). As a result, the windows are non-maximal with respect to being open.

However, a statement that some of the windows are unbroken is compatible both with a situation requiring repairs, and also with a situation not requiring them, leaving the decision problem completely unresolved (62d). 
Sophia A. Malamud

(62)

d. Pool of propositions for Pred $_{2}$ :

*open $\left(\uparrow \sigma x^{*}\right.$ window $\left.(x, w)\right) \&$ *unbr $\left(d_{1}\right)$

no actions eliminated

*open $(\uparrow \sigma x *$ window $(x, w)) \&$ *unbr $\left(d_{2}\right)$

no actions eliminated

*open $(\uparrow \sigma x *$ window $(x, w)) \&$ *unbr $\left(d_{1} \sqcup d_{2}\right) \quad$ eliminates $a_{1}$ and $a_{3}$ Thus, $\operatorname{Rel}(\operatorname{DeP})\left(I P_{2 \theta_{2}}\right)(w)=\left\{d_{1} \sqcup d_{2}\right\}$

Only propositions in which all windows are unbroken tell us that no repairs are needed - this is the optimally relevant option reflected in each of the resulting propositions in (62e). As a result, the windows are maximal with respect to being unbroken. ${ }^{24}$

e. $\llbracket I P^{t o p} \rrbracket=\lambda w \exists p[p \in\{$

$\lambda w$. *open $\left(e_{1}, w\right) \& * \theta_{1}\left(e_{1}\right)=d_{1} \& * \operatorname{unbr}\left(e_{2}, w\right) \& * \theta_{2}\left(e_{2}\right)=d_{1} \sqcup d_{2}$, $\lambda w . *$ open $\left(e_{1}, w\right) \& * \theta_{1}\left(e_{1}\right)=d_{2} \& * \operatorname{unbr}\left(e_{2}, w\right) \& * \theta_{2}\left(e_{2}\right)=d_{1} \sqcup d_{2}$, $\lambda w . * \operatorname{open}\left(e_{1}, w\right) \& * \theta_{1}\left(e_{1}\right)=d_{1} \sqcup d_{2} \&$ $\left.\left.* u n b r\left(e_{2}, w\right) \& * \theta_{2}\left(e_{2}\right)=d_{1} \sqcup d_{2}\right\} \& p(w)\right]$

The sets of windows resulting from the action of $\mathrm{Rel}$ on $(62 \mathrm{C}, \mathrm{d})$ are then used in constructing the set of optimally relevant propositions, according to the definition in (61), with the elements of the set of windows most relevant for open assigned a theta-role by open, and the windows from the set most relevant for unbroken assigned a theta-role by unbroken. The resulting propositions are therefore optimally relevant with respect to both predicates; the existential at the top turns this into a single proposition stating that at least one of the propositions in the set is true (62e).

Finally, combining negation and predicate coordination, consider (63a), in the context of the decision problem in EMPTY HOUSE (59a). On the reading where negation has scope over conjunction, the sentence has the same interpretation as the one in (63b), since English obeys DeMorgan's law. Note that the earlier system (51), in which the propositions in the pool evaluated by REL were "local" in the sense of including just the NP and the predicate, would encounter a scopal paradox in this reading: on the one hand, negation scopes over the conjunction of the two predicates, while on the other hand, the negation must be included in the relevance calculation for each conjunct.

24 Note that while the individual REL calculations in (62c) and (62d) fail to resolve the decision problem fully, the ultimately constructed interpretation does. The sentence in context does not generate any ignorance/indifference implicatures, e.g., that the speaker does not know what the optimal action is (I am grateful to an anonymous reviewer for this observation). The absence of the implicature suggests that the pragmatic calculation in REL is separate from the more "global" pragmatic calculations, such as the computation of implicatures. 
The meaning of plural definites

The proposal in (58) in which the entire utterance is considered avoids this problem.

(63) Situation: as in EMPTY HOUSE (59a)

a. Negating coordinated predicates

The windows aren't open and unbroken.

b. The windows are closed or broken.

The derivation for the negation-over-conjunction reading is very similar to the one in (62), and includes the negation in calculating the most relevant windows (64).

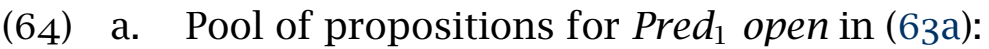

$\neg *$ open $\left(d_{1}\right) \vee \neg^{*} \operatorname{unbr}\left(\uparrow \sigma x^{*}\right.$ window $\left.(x, w)\right) \quad$ no eliminations

$\neg$ *open $\left(d_{2}\right) \vee \neg *$ unbr $\left(\uparrow \sigma x^{*}\right.$ window $\left.(x, w)\right) \quad$ no eliminations

$\neg$ *open $\left(d_{1} \sqcup d_{2}\right) \vee \neg * u n b r\left(\uparrow \sigma x^{*}\right.$ window $\left.(x, w)\right) \quad$ eliminates $a_{4}$

Thus, $\operatorname{Rel}(\operatorname{DeP})\left(I P_{1 \theta_{1}}\right)(w)=\left\{d_{1} \sqcup d_{2}\right\}$

b. Pool of propositions for Pred 2 unbroken in (63a):

$\neg$ open $\left(\uparrow \sigma x^{*}\right.$ window $\left.(x, w)\right) \vee \neg * u n b r\left(d_{1}\right)$

no eliminations

$\neg$ *open $\left(\uparrow \sigma x^{*}\right.$ window $\left.(x, w)\right) \vee \neg * u n b r\left(d_{2}\right)$ no eliminations

$\neg$ *open $\left(\uparrow \sigma x^{*}\right.$ window $\left.(x, w)\right) \vee \neg * u n b r\left(d_{1} \sqcup d_{2}\right) \quad$ no eliminations Thus, $\operatorname{Rel}(\operatorname{DeP})\left(I P_{2 \theta_{2}}\right)(w)=\left\{d_{1}, d_{2}, d_{1} \sqcup d_{2}\right\}$

c. $\llbracket(63 a) \rrbracket=\lambda w \exists p[p \in\{$

$\lambda w . \neg\left[{ }^{*} \operatorname{open}\left(e_{1}, w\right) \& * \theta_{1}\left(e_{1}\right)=d_{1} \sqcup d_{2} \& * \operatorname{unbr}\left(e_{2}, w\right) \& * \theta_{2}\left(e_{2}\right)=\right.$ $\left.d_{1}\right]$

$\lambda w . \neg\left[* \operatorname{open}\left(e_{1}, w\right) \& * \theta_{1}\left(e_{1}\right)=d_{1} \sqcup d_{2} \& * \operatorname{unbr}\left(e_{2}, w\right) \& * \theta_{2}\left(e_{2}\right)=\right.$ $\left.d_{2}\right]$,

$\lambda w . \neg\left[* \operatorname{open}\left(e_{1}, w\right) \& * \theta_{1}\left(e_{1}\right)=d_{1} \sqcup d_{2} \& * \operatorname{unbroken}\left(e_{2}, w\right) \& * \theta_{2}\left(e_{2}\right)=\right.$ $\left.\left.\left.d_{1} \sqcup d_{2}\right]\right\} \& p(w)\right]$

Predicates distribute over sums, so the calculation in (64) correctly predicts that, on the negation-over-conjunction reading, both of the windows aren't open, or else that one or both of the window's aren't unbroken - that is, that both windows are closed, or one or both are broken.

\subsubsection{Multiple vague NPs}

This proposal has a natural extension to cases involving underspecified definite plural NPs in two different roles. When underspecified NPs are 
present in multiple positions, we can form tuples of NP denotations, and calculate the reading that is maximally relevant with respect to all NPs (65).

(65) The relevance operator (extended second version)

$$
\begin{aligned}
& R E L(D e P)\left(X_{n \theta_{n}^{i} \theta_{n}^{j}}\right)(w)=\quad \text { For predicates with two } \\
& \left\{\left(g_{i}, g_{j}\right): \sqcup\left\{\downarrow d: d \in A T\left(\downarrow g_{i}\right)\right\} \subseteq \downarrow \llbracket N P_{\theta_{n}^{i}} \rrbracket(w) \quad\right. \text { NPs, REL forms pairs } \\
& \& \sqcup\left\{\downarrow d: d \in A T\left(\downarrow g_{j}\right)\right\} \subseteq \downarrow \llbracket N P_{\theta_{n}^{j}} \rrbracket(w) \quad \text { of NP-made individuals, } \\
& \& \neg \exists h_{i} \exists h_{j}\left[\sqcup\left\{\downarrow d: d \in A T\left(\downarrow h_{i}\right)\right\} \subseteq \downarrow \llbracket N P_{\theta_{n}^{i}} \rrbracket(w)\right. \text { such that no other pair } \\
& \& \sqcup\left\{\downarrow d: d \in A T\left(\downarrow h_{j}\right)\right\} \subseteq \downarrow \llbracket N P_{\theta_{n}^{j}} \rrbracket(w) \quad \text { of NP-made individuals } \\
& \&\left[\lambda w \cdot \llbracket X_{n \theta_{n}^{i} \theta_{n}^{j}} \rrbracket\left[h_{i} / \llbracket N P_{\theta_{n}^{i}} \rrbracket, h_{j} / \llbracket N P_{\theta_{n}^{j}} \rrbracket\right]>_{D e P} \quad\right. \text { would form more } \\
& \left.\left.\left.>_{D e P} \lambda w \cdot \llbracket X_{n \theta_{n}^{i} \theta_{n}^{j}} \rrbracket\left[g_{i} / \llbracket N P_{\theta_{n}^{i}} \rrbracket, g_{j} / \llbracket N P_{\theta_{n}^{j}} \rrbracket\right]\right]\right]\right\} \quad \text { relevant propositions }
\end{aligned}
$$

The $O_{R e l}$ operator then goes through this set of pairs, matching the optimal subparts of the two NPs to the appropriate theta-roles (66).

$$
\begin{aligned}
& O_{R e l}^{n \theta_{1}^{i} \theta_{n}^{j}}=\lambda w \cdot \lambda \phi . \lambda x_{i} . \lambda x_{j} . \lambda e . \phi=\llbracket X_{n \theta_{n}^{i} \theta_{n}^{j}} \rrbracket \& \\
& \left(x_{i}, x_{j}\right) \in R E L(D e P)\left(X_{n \theta_{n}^{i} \theta_{n}^{j}}\right)(w) \& \\
& \llbracket X \rrbracket\left[x_{i} / \llbracket N P_{\theta_{n}^{i}} \rrbracket, x_{j} / \llbracket N P_{\theta_{n}^{j}}^{j} \rrbracket\right] \\
& =\lambda w \cdot \lambda \phi \cdot \lambda x_{i} \cdot \lambda x_{j} \cdot \lambda e \cdot \phi=\llbracket X_{n \theta_{n}^{i} \theta_{n}^{j}} \rrbracket \& \\
& \left(x_{i}, x_{j}\right) \in \operatorname{REL}(\operatorname{DeP})\left(X_{n \theta_{n}^{i} \theta_{n}^{j}}\right)(w) \\
& \text { spelling out the contents } \\
& \text { of the reconstituted } \\
& \&\left[\ldots \llbracket \text { Predicate }_{n} \rrbracket(e, w) \& * \theta_{n}^{i}(e)=x_{i} \& * \theta_{n}^{j}(e)=x_{j} \ldots\right] \text { proposition }
\end{aligned}
$$

For example, if a journalistic team of students $\mathrm{Al}$ and Bob interview two award-winning professors C. and D. for a newspaper, the reading in (67) is non-maximal with respect to students, and maximal with regard to professors.

(67) a. The students interviewed the professors.

b.

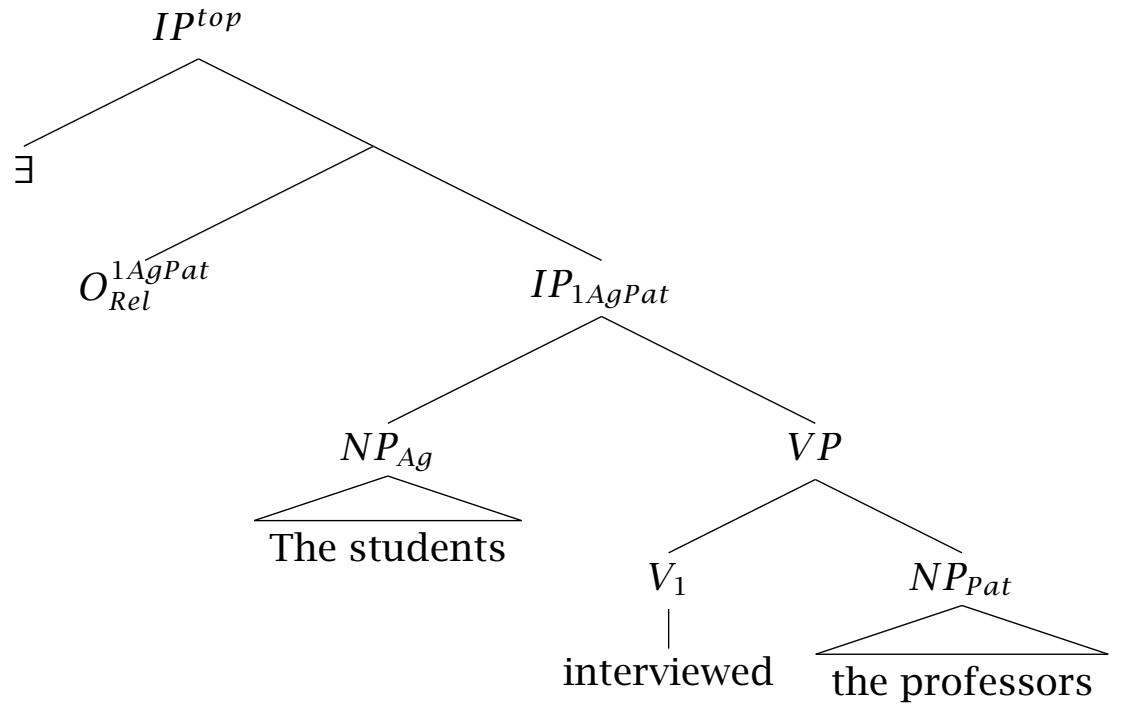


The meaning of plural definites

The optimal interviewer-interviewee pairs emerge as in (68).

$$
\begin{aligned}
& R E L(D e P)\left(I P_{1 \text { AgPat }}\right)(w)=\{(a, c \sqcup d),(b, c \sqcup d),(a \sqcup b, c \sqcup d),(\uparrow(a \sqcup \\
& b), c \sqcup d)\}
\end{aligned}
$$

The definition in (66) yields the propositions in (69), generating the desired reading.

(69) $\llbracket(67 \mathrm{a}) \rrbracket=\lambda w \exists p[p \in\{$

$\lambda w$. *interviewed $(e, w) \& * A g(e)=a \& * \operatorname{Pat}(e)=c \sqcup d]$,

$\lambda w$. *interviewed $(e, w) \& * A g(e)=b \& * \operatorname{Pat}(e)=c \sqcup d]$,

$\lambda w$. *interviewed $(e, w) \& * A g(e)=a \sqcup b \& * \operatorname{Pat}(e)=c \sqcup d]$,

$\lambda w . *$ interviewed $(e, w) \& * A g(e)=\uparrow(a \sqcup b) \& * \operatorname{Pat}(e)=c \sqcup d]\} \& p(w)]$

This system is not sufficient for situations in which, for instance, $\mathrm{Al}$ and $C$. are in the math department, while Bob and D. are in the linguistics department, and the students must interview professors in their respective departments. In order to generate such readings, each student-professor pair has to be tied to a particular subevent of the plural interviewing event, in the way that subparts of NPs are tied to subparts of events in the derivations in Landman (1996) (\$2.3). I leave this to future work, and turn to the discussion of theoretical consequences of this proposal.

\section{Discussion and further issues}

\subsection{Comparison with prior approaches to variability in definite plurals.}

The present proposal does at least as well as competing explanations, the Homogeneity Presupposition, and the Strongest Meaning Hypothesis, for those cases that these approaches handle well, and additionally is able to derive correct interpretations for cases where the HP and the SMH fail. I first briefly review the HP and the SMH and discuss ways in which the present proposal achieves broader empirical coverage, and then turn to the data that the SMH handles well, and discuss the performance of my proposal for these cases.

\subsubsection{Cases for which the present proposal does better than the HP and the SMH}

The Homogeneity Presupposition, a version of which is given in (70), has been used by Schwarzschild (1994) and Löbner $(1985,1987,2000)$ to explain the 
data in (1), and extended by Szabolcsi \& Haddican (2004) to apply to definite conjunctions.

(70) $G$ is a plurality, * $P$ is a pluralised, and therefore a distributive predicate. (Beck 2001)

$$
* P(G)=\left\{\begin{array}{l}
1 \text { if } \forall x(x \in G \rightarrow P(x)) \\
0 \text { if } \forall x(x \in G \rightarrow \neg P(x)) \\
\text { undefined otherwise }
\end{array}\right.
$$

Beck also generalises this definition (70) to two-place predicates, as in (1).

(1) LINGUISTS

a. Peter didn't see the linguists.

(not any of the linguists)

b. Peter saw the linguists.

(all of the linguists)

Given the homogeneity presupposition, the not any of the linguists reading of (1a) is simply a matter of the plurality of linguists undergoing homogeneous distributive predication in the scope of negation. Thus, the presupposition that Peter saw $x$ applies to all the linguists or none of them, together with (1a), comes to mean that Peter failed to see any of the linguists.

Downward-entailing and NPI-licensing contexts other than negation also show the same interpretation preferences (71, from Breheny 2005).

(71) If Peter beats the donkeys in his care, he will be prosecuted.

(prosecution for beating any donkey)

According to the HP, Peter beats the donkeys in his care presupposes that all of the donkeys get beaten or none of them. Presuppositions of the antecedent project; therefore the entire sentence should presuppose that either all or none of Peter's donkeys are beaten. Together with the assertion, this homogeneity presupposition derives the preferred reading of (71), in which Peter is prosecuted as soon as he beats at least one of his donkeys. ${ }^{25}$ However, note that the HP does not behave like a presupposition in this case - the sentence is not constrained to contexts in which Peter beats all or none of his donkeys, but can be used felicitously in contexts in which he only beats some.

Accounts that depend on the HP run into several problems. First, Breheny (2005) points out that in examples like (72), there seems to be no expectation

25 I am grateful to an anonymous reviewer for suggesting this interpretation of the HP for this sentence. 
The meaning of plural definites

that capturing the gangsters is an all-or-nothing matter, making homogeneity an implausible explanation for the preferred reading that the police have, as of yet, caught none of them.

(72) The gang members have dispersed to the four corners of the world after the bank robbery, and the police haven't found them yet.

Second, the preference for maximal and non-maximal readings is preserved in contexts in which the homogeneity is directly denied. For instance, the sentence in (71) can be used in contexts in which the homogeneity is explicitly contradicted $\left(71^{\prime}\right)$. In such contexts, it still follows that Peter will be prosecuted if he beats any of his donkeys.

$\left(71^{\prime}\right)$ Ann: Peter treats all his donkeys except Gray very well, but he beats poor Gray.

Bob: We can report him to the authorities: if Peter beats the donkeys in his care, he will be prosecuted.

The dialogue in $\left(71^{\prime}\right)$ highlights the fact that the preferred reading of (71) does not depend on homogeneity. Consequently, the Homogeneity Presupposition cannot possibly derive the interpretational preferences in (1, 71).

Finally, the Homogeneity Presupposition fails to derive non-maximal readings in upward-entailing contexts, such as SAFE HOUSE (2b), BANK DOORS (8b), DunEs (4), and BEGGARS (5). These reasons lead us to abandon the HP.

An alternative to the HP is the Strongest Meaning Hypothesis, a competing explanation for the maximality variation in definite plurals. Krifka (1996b) proposes that, when a predicate applies to any sum individual, the grammar does not specify how many subparts of the individual need to actually satisfy the predicate, leaving the semantics underdetermined. Then, the logically strongest available reading is chosen pragmatically (the Strongest Meaning Hypothesis, or SMH, 73) (Dalrymple, Kanazawa, Kim, et al. 1998; see also Beck 2001).

(73) The readings of semantically underspecified sentences are ordered by entailment relation. The strongest reading (one that entails all others) is preferred to others.

The SMH immediately explains why downward-entailing contexts favour the existential, and upward-entailing contexts the universal, readings.

For example, in (71), the semantics leaves the conditional underdetermined: Peter could be facing prosecution for beating some of the donkeys, or 
all of the donkeys. However, if he's prosecuted for beating only some of the donkeys, he will be all the more so for beating all of them: the non-maximal "any" reading entails the "all" reading. Therefore, this non-maximal reading is pragmatically preferred, according to the $\mathrm{SMH}$.

Downward-entailing contexts will favour the non-maximal (any) reading, while upward-entailing contexts will result in the maximal (all) reading for all predicates applying to sum individuals.

The SMH fails to account for the context-dependent variability in definite plurals, as demonstrated by examples in which the logically weaker reading prevails, such as SAFE HOUSE (2b) and BANK DOORS (8b). The main reason for this failure is that it is not the strongest, but rather the most relevant readings that are pragmatically chosen for underspecified utterances. As a result, the SMH explains the tendencies exemplified in LINGUISTS (1), but is too strong a condition, predicting these tendencies to be absolute.

\subsubsection{From relevance to strength: cases that the SMH handles well ${ }^{25}$}

The SMH and my proposal appear to suffer from opposite problems: the SMH explains the change of preferences from maximal to non-maximal in upwardand downward-entailing contexts, but is not sensitive to other features of the context, such as packaging or hearer goals. At first blush, it would seem that a purely pragmatic proposal, such as mine, while able to account for the influence of extra-linguistic context, cannot handle the tendencies in LINGUISTS (1).

Note, however, that these cases motivating the SMH are sentences presented in isolation, rather than within a discourse. In such contexts (or rather, without explicit context), the truth-conditional content of the utterance takes center stage - hearers prefer to have as much information as possible, in effect defaulting to scenarios in which truth matters most. In the absence of a contextually provided QUD or DeP, the hearer's choice of actions is simply the choice of various mental updates: the QUD is What is the world

25 I am very grateful to two anonymous reviewers whose comments led to the writing of this section. One reviewer pointed out that my account needs to explain the central tendencies exemplified in LINGUISTS (1) and captured by the SMH - that out-of-the-blue upward-entailing contexts favour maximal interpretations, and downward-entailing contexts favour nonmaximal readings. The other reviewer suggested that I include a section connecting relevance and strength based on the discussion in van Rooij (2004), and articulated the claim that my "relevance-based approach conservatively subsumes the 'strongest-meaning-hypothesis'approach". 
The meaning of plural definites

like? These different mental update actions can be thought of as probability distributions over $W$, representing beliefs - "sometimes inferences, or statements of belief, may be regarded as ends in themselves, to be judged independently of any "practical' decision problem" (van Rooij 2004: p. 393). Under-informative, underspecified utterances cannot be "good enough" in helping the hearer choose, because they do not resolve the QUD What is the world like?.

Such fact-finding contexts support utility functions different from $U_{01}$, which we have used so far. Following Bernardo (1979), van Rooij (2004) proves that fact-finding is facilitated by payoffs that depend on the informational value of a proposition, defined as in (74).

(74) a. Shannon Entropy $E(Q)=\sum_{q \in Q} P(q) \log _{2} \frac{1}{P(q)}$

$$
=\sum_{q \in Q} P(q) \times\left(-\log _{2} P(q)\right)
$$

b. Informational Value $I V(m)=E(Q)-E(Q \mid m)$

$$
=\sum_{q \in Q} P(q) \times\left(-\log _{2} P(q)\right)-\sum_{q \in Q} P(q \mid m) \times\left(-\log _{2} P(q \mid m)\right)
$$

The Shannon entropy (which Turing called the weight of evidence) of a set of messages is the mean information content per message (74) (cf. e.g. Cover \& Thomas 1991). The negative log function gives greater values to less probable (less predictable, more surprising) messages. The entropy of a question thus measures how much information can be learned (on average) by getting an answer. The informational value of a proposition can be gauged by looking at how much less useful it makes the question - to what extent the answer to the question becomes predictable.

Bernardo (1979) uses (74), demonstrating that a utility function that encourages the speaker to truthfully report the maximum amount of information must be of the form $A \times \log P(m)+B$, where $A$ is a positive constant, and $B$ an arbitrary parameter (75a). Van Rooij (2004) demonstrates that this logarithmic utility function reduces the utility value of a proposition to its informational value (75b). As a result, in contexts in which the participants are concerned with the truth, the utility-based ordering in (47) reduces to informativity (Bernardo 1979, van Rooij 2004, Shannon 1948) (74). 
(75) a. Utility function for fact-finding contexts

$U_{\log }=U V(P, m)=A \times \log P(m)+B$

b. Utility value of learning $m$ in a situation where QUD is $W$

$U V(m)=U V$ (learn $m$, then choose update) $-U V$ (choose now)

$=\sum_{w \in W} P(w \mid m) \times \log P(w \mid m)-\sum_{w \in W} P(w) \times \log P(w)$

$=-\sum_{w \in W} P(w \mid m) \times(-\log P(w \mid m))+\sum_{w \in W} P(w) \times(-\log P(w))=$ $I V(m)$

If we further assume that when a proposition is learned, all cells of the QUD consistent with it have equal probability, this informativity-based ordering reduces to semantic entailment in contexts where the QUD is $W$, What is the world like? (cf. van Rooij 2004). As a consequence, speakers and hearers in fact-finding contexts will conform to the $\mathrm{SMH}$ - semantically stronger propositions are also the most relevant ones.

In light of this discussion, the present proposal can be seen as a generalisation of the SMH. Speakers guess at hearers' utility functions based on the context - a dilemma such as the one in SAFE WINDOws leads the speaker to choose $U_{01}$, while in the context of a Martian geology report or a context-less sentence such as LINGUISTS, the speaker will guess that $U_{\log }$ is the best bet. The decision-theoretic approach will, in the latter case, replicate the results of the $\mathrm{SMH}$, deriving the strength-based interpretation preferences demonstrated in LINGUISTS (1). At the same time, when contextual factors support a less truth-based utility function, my proposal will perform better than the SMH, deriving the goal-based interpretations exemplified in SAFE HOUSE (2).

\section{Conclusion}

This paper proposes a model of interpretation in which the precise meaning of utterances is derived from underspecified semantics and contextual influences. The DT approach takes as its base a weak semantics for definite plurals, and builds in pragmatic factors to derive stronger truth-conditions for sentences with definites. Speakers' estimates of each other's beliefs, goals, and available actions are incorporated into the selection of maximally relevant propositions with subparts of the plural definite. The framework derives the (non)maximality and distributivity patterns described and makes correct predictions about speaker knowledge. 
The meaning of plural definites

The proposal is implemented by positing a pragmatic relevance operator in the object language. However, this is a technical solution only; the operator is not necessary to capture decision-theoretic optimisation. As a first step towards an implementation that does not include such an operator, note that most of the work is done by the indexing, allowing the information about vague NP-predicate pairs to percolate up to the relevance operator. If we assume that this same information is available at the conclusion of compositional calculations, we can invoke an automatic process whereby optimally relevant propositions are chosen without any relevance operators in the syntactic tree. In this case, REL becomes a description of a semantic/pragmatic operation, akin to the exhaustivity operator invoked by Groenendijk \& Stokhof (1984) and related work. I believe that this crucially distinguishes my proposal from the recent claims that covert object-language exhaustivity operators are necessary in the analysis of (some) implicatures (Chierchia 2004, Fox 2007, Landman 2000, inter alia).

A central feature of my analysis is the compositional derivation of competing interpretations. The goals of the hearer (as guessed by the speaker) give rise to a ranking of these interpretational alternatives. The ultimate interpretation is essentially a disjunction of the optimally helpful alternatives. In this, my analysis departs from the traditional Gricean approach, in which it is alternative potential utterances, rather than alternative readings of a single utterance, that are considered. ${ }^{26}$ In this, my proposal is similar to that of Parikh (2000), who considers competing interpretations of an utterance in a game-theoretic account of ambiguity resolution.

In contexts in which truth is of paramount importance, the proposed procedure reduces to the Strongest Meaning Hypothesis, deriving the preference for maximal readings in upward-entailing and non-maximal readings in downward-entailing contexts. This is because the utility ranking for propositions in such contexts comes down to the informativity of the propositions.

The reasoning which allows the hearer to calculate the correct interpretation is particularised (Grice 1975b) - it is specific to the particular situations of use. In this, my proposal resembles those of Benz (2006), Benz \& van Rooij (2007), Parikh (1992, 2000). In these game-theoretic accounts of implicature and question-answering, the authors present a view of pragmatic calculation in which the hearer is choosing between alternative interpretations (as in my proposal), while the speaker is choosing between alternative utterances.

26 I thank an anonymous reviewer for pointing this out. 
For instance, in Benz \& van Rooij's (2007) analysis of questions and answers, the answerers address the decision problems of the questioners, while at the same time maximising their own utilities. The payoff functions for the hearer and speaker are nearly identical (differing by a "nominal" cost of sending a message), capturing the spirit of the Cooperative Principle. This alignment of utilities means that the utility of the various alternative answers depends on how it influences the resolution of the hearer's problem. In effect, then, the hearer can count on the speaker to produce an optimally relevant answer because this answer would have the highest utility for the speaker.

My proposal takes a simplified view of communication in comparison to the game-theoretic framework set out in Benz \& van Rooij (2007), since it does not represent the speaker's decision problem of choosing among alternative utterances. ${ }^{27}$ This simplification is made possible by two assumptions. First, while I refer to "the hearer's decision problem", that problem is reinterpreted as the QUD in the conversation. I assume that each declarative utterance in discourse "answers" a QUD. Unlike the question-answering dialogues in Benz \& van Rooij (2007), nobody actually asks the QUD in most cases; however, speakers and hearers share the goal of addressing the mutually-estimated QUD. Second, the speaker's choice of utterance is taken for granted, and assumed to be maximally helpful to the hearer. This assumption of optimal relevance can be thought of as a consequence of aligned speaker and hearer preferences, as in Benz \& van Rooij (2007).

While it does not provide sufficient conditions for the use of underspecified utterances, this simplification makes it possible for us to concentrate on the hearer's decision problem of choosing among alternative interpretations, and set aside the important issues of the speaker's decision between linguistic forms and of the different ways that QUDs arise and are negotiated in conversation. Consequently, we are able to examine the influence of the QUD and the hearer's goals on the interpretation of underspecified utterances. In fact, the proposed procedure suggests a necessary condition under which the speaker will choose a particular underspecified form - when this form is "good enough" for resolving the QUD. ${ }^{28}$ This condition was already suggested by Grice, who gives (76) as an example of such an imprecise, yet optimal, utterance (Grice 1975a: p. 40).

27 My view does not incorporate the speaker's goals in the sense that it does not represent the speaker's decision problem of choosing the optimal utterance-I am grateful to an anonymous reviewer for raising this issue.

28 I am grateful to Anton Benz and Robin Clark for discussing this issue with me. 
The meaning of plural definites

(76) Situation: It is generally known that New York and Boston were blacked out last night. A asks B whether C saw a particular TV program last night. B knows that $\mathrm{C}$ was in New York.

Optimal utterance: No, he was in a blacked-out city.

B could have been more precise, but the optimal utterance resolves both the immediate question posed, and also an additional implicit QUD raised by the answer No - why C didn't see the program.

I expect the model of interpretation proposed here to generalise to other cases in which semantically underspecified expressions acquire more precise meanings in context. Such interpretive phenomena as domain selection for definites and quantifiers, pronoun resolution, and calculation of indirect speech acts all exhibit variability based on the goals of speakers and hearers in discourse. Once the competing alternative interpretations are calculated, the final meaning will be the optimal one relative to the QUD. This means that in cases when multiple alternatives are equally optimal, a degree of underspecification will remain. In the case of sentences with definite plurals, the source of competing alternatives is compositional semantics; the presence of underspecified plurals signals that relevance-based reasoning will be needed to arrive at the final interpretation. This study can thus be seen as a step towards a better understanding of the role of strategic reasoning in the overall division of pragmatic labour.

\section{References}

Barker, Chris. 1996. Presuppositions for proportional quantifiers. Natural Language Semantics 4(3). 237-259. http://dx.doi.org/10.1007/BFo0372821. Beck, Sigrid. 2001. Reciprocals are definites. Natural Language Semantics 9(1). 69-138. http://dx.doi.org/10.1023/A:1012203407127.

Beck, Sigrid \& Hotze Rullmann. 1999. A flexible approach to exhaustivity in questions. Natural Language Semantics 7(3). 249-298. http://dx.doi.org/ 10.1023/A:1008373224343.

Benz, Anton. 2006. Utility and relevance of answers. In Anton Benz, Gerhard Jäger \& Robert van Rooij (eds.), Game Theory and Pragmatics, 195-219. Palgrave Macmillan.

Benz, Anton \& Robert van Rooij. 2007. Optimal assertions and what they implicate. Topoi 26(1). 63-78. http://dx.doi.org/10.1007/s11245-006-90073. 
Berman, Stephen. 1991. On the Semantics and Logical Form of Wh-Clauses. Amherst, MA: University of Massachusetts PhD thesis.

Bernardo, José M. 1979. Expected Information as Expected Utility. The Annals of Statistics 7(3). 686-690. http://dx.doi.org/10.1214/aos/1176344689.

Breheny, Richard. 2005. Exhaustivity, homogeneity and definiteness. The Fifteenth Amsterdam Colloquium. 59-65. http://www.illc.uva.nl/AC/ AC2005/uploaded_files/ACo5Proceedings.pdf.

Brisson, Christine. 1998. Distributivity, Maximality, and Floating Quantifiers. Rutgers University PhD thesis.

Chierchia, Gennaro. 2004. Scalar implicatures, polarity phenomena, and the syntax/pragmatics interface. In Adriana Belletti (ed.), Structures and beyond: The cartography of syntactic structures, vol. 3, 39-103. Oxford: Oxford University Press.

Cover, Thomas M. \& Joy A. Thomas. 1991. Elements of information theory. New York: Wiley Interscience.

Dalrymple, Mary, Makoto Kanazawa, Yookyung Kim, Sam A. Mchombo \& Stanley Peters. 1998. Reciprocal expressions and the concept of reciprocity. Linguistics and Philosophy 21(2). 159-210. http://dx.doi.org/10.1023/A: 1005330227480.

Dalrymple, Mary, Makoto Kanazawa, Sam A. Mchombo \& Stanley Peters. 1994. What do reciprocals mean? Proceedings of Semantics and Linguistic Theory 4. $61-78$.

Dowty, David. 1987. Collective predicates, distributive predicates, and all. The Third Eastern States Conference on Linguistics (ESCOL3). 97-115.

von Fintel, Kai. 1997. Bare plurals, bare conditionals, and only. Journal of Semantics 14(1). 1-56. http://dx.doi.org/10.1093/jos/14.1.1.

Fodor, Janet Dean. 1970. The Linguistic Description of Opaque Contexts. MIT $\mathrm{PhD}$ thesis.

Fox, Danny. 2007. Free choice and the theory of scalar implicatures. In Uli Sauerland \& Penka Stateva (eds.), Presupposition and implicature in compositional semantics, 71-120. Hampshire: Palgrave Macmillan.

Franke, Michael. 2011. Quantity implicatures, exhaustive interpretation, and rational conversation. Semantics and Pragmatics 4(1). 1-82. http://dx.doi. org/10.3765/sp.4.1.

Geurts, Bart \& Nausicaa Pouscoulous. 2009. Embedded implicatures?!? Semantics and Pragmatics 2(4). 1-34. http://dx.doi.org/10.3765/sp.2.4. 
The meaning of plural definites

Grice, H. Paul. 1975a. Further notes on logic and conversation. In Peter Cole \& Jerry L. Morgan (eds.), Syntax and Semantics. vol. III. Speech Acts. New York: Academic Press.

Grice, H. Paul. 1975b. Logic and conversation. In Peter Cole \& Jerry L. Morgan (eds.), Syntax and Semantics. vol. III. Speech Acts. 41-58. New York: Academic Press.

Groenendijk, Jeroen \& Martin Stokhof. 1984. Studies on the Semantics of Questions and the Pragmatics of Answers. Amsterdam: University of Amsterdam PhD thesis.

Heim, Irene. 1994. Puzzling reflexive pronouns in de se reports. Unpublished Handout. http:/ / semanticsarchive.net/Archive/mVkNjZlY / Bielefeld\% 2ohandout\%2094.pdf.

Horn, Laurence R. 1984. Toward a new taxonomy for pragmatic inference: Qbased and R-based implicatures. In Deborah Schiffrin (ed.), Meaning, Form, and Use in Context. 11-42. Washington: Georgetown University Press.

Hulstijn, Joris. 1997. Structured information states: Raising and resolving issues. MunDial97. 99-117. http://homepage.tudelft.nl/w98h5/Articles/ mundial.pdf.

Kennedy, Christopher. 2007. Vagueness and grammar: The semantics of relative and absolute gradable adjectives. Linguistics and Philosophy 30(1). 1-45. http://dx.doi.org/10.1007/s10988-006-9008-o.

Kratzer, Angelika. forthcoming. Cumulativity as a possible universal. In The Event Argument. MIT Press.

Kratzer, Angelika \& Junko Shimoyama. 2002. Indeterminate pronouns: The view from Japanese. The Tokyo Conference on Psycholinguistics 3. 1-25.

Krifka, Manfred. 1996a. Parametrized sum individuals for plural anaphora. Linguistics and Philosophy 19(6). 555-598. http://dx.doi.org/10.1007/ BFoo632708.

Krifka, Manfred. 1996b. Pragmatic strengthening in plural predications and donkey sentences. Semantics and Linguistic Theory (SALT VI). 136-153.

van Kuppevelt, Jan. 1995. Discourse structure, topicality and questioning. Journal of Linguistics 31(1). 109-147. http://dx.doi.org/10.1017/So02222670000058X.

Lahiri, Utpal. 1991. Embedded Interrogatives and the Predicates that Embed Them. MIT PhD thesis. http://dx.doi.org/1721.1/13520.

Landman, Fred. 1989. Groups, I. Linguistics and Philosophy 12(5). 559-605. http://dx.doi.org/10.1007/BFoo627774.

Landman, Fred. 1996. Plurality. In Shalom Lappin (ed.), The Handbook of Contemporary Semantic Theory, 425-451. Blackwell Publishing. 
Landman, Fred. 200o. Events and plurality: The Jerusalem lectures. Dordrecht: Kluwer Academic Publishers.

Lappin, Shalom. 1989. Donkey pronouns unbound. Theoretical Linguistics 15(3). 263-289. http://dx.doi.org/10.1515/thli.1988.15.3.263.

Link, Godehard. 1983. The logical analysis of plurals and mass terms: A lattice-theoretical approach. In Rainer Bäuerle, Christoph Schwarze \& Arnim von Stechow (eds.), Meaning, Use and Interpretation of Language, 302-323. De Gruyter.

Löbner, Sebastian. 1985. Definites. Journal of Semantics 4(4). 279-326. http: //dx.doi.org/10.1093/jos/4.4.279.

Löbner, Sebastian. 1987. The conceptual nature of natural language quantification. The '87 Debrecen Symposium on Logic and Language. 81-94.

Löbner, Sebastian. 2000. Polarity in natural language: Predication, quantification and negation in particular and characterizing sentences. Linguistics and Philosophy 23(3). 213-308. http://dx.doi.org/10.1023/A: 1005571202592.

Merin, Arthur. 1999. Information, relevance, and social decisionmaking: Some principles and results of decision-theoretic semantics. In Lawrence S. Moss, Jonathan Ginzburg \& Maarten de Rijke (eds.), Logic, language, and computation, vol. 2, 179-221. Stanford: CSLI.

Parikh, Prashant. 1992. A game-theoretic account of implicature. The 4th Conference on Theoretical Aspects of Reasoning about Knowledge (TARK 4). 85-94. http://portal.acm.org/citation.cfm?id=1029762.1029769.

Parikh, Prashant. 2000. Communication, Meaning, and Interpretation. Linguistics and Philosophy 23(2). 185-212. http://dx.doi.org/10.1023/A: 1005513919392.

Parsons, Terence. 1990. Events in the Semantics of English: A Study in Subatomic Semantics. Cambridge, Massachusetts: MIT Press.

Potts, Christopher. 2006. Conversational implicatures via general pragmatic pressures. In Eric McCready (ed.), Logic Engineering and Natural Language Semantics 2006, 65-79. Tokyo: Japanese Society for Artificial Intelligence. http://semanticsarchive.net/Archive/mI1N2YzM/potts-lenlso6pragmatic-pressures.pdf.

Roberts, Craige. 1996. Information structure: Towards an integrated formal theory of pragmatics. In Jae Hak Yoon \& Andreas Kathol (eds.), OSU Working Papers in Linguistics 49: Papers in Semantics, 91-136. Columbus, OH: The Ohio State University Department of Linguistics. http://www. ling.ohio-state.edu/ croberts/infostr.pdf. 
The meaning of plural definites

van Rooij, Robert. 1999. Questioning to resolve decision problems. The Twelfth Amsterdam Colloquium.

van Rooij, Robert. 2003. Questioning to resolve decision problems. Linguistics and Philosophy 29(6). 727-763. http:// dx.doi.org/10.1023/B:LING . oooooo4548.98658.8f.

van Rooij, Robert. 2004. Utility, informativity and protocols. Journal of Philosophical Logic 33(4). 389-419. http:// dx. doi . org/10.1023/B:LOGI . ooooo36830.62877.ee.

van Rooij, Robert. 2011. Optimality-Theoretic and Game-Theoretic approaches to implicature. In Edward N. Zalta (ed.), The Stanford Encyclopedia of Philosophy, Spring 2011. http://plato.stanford.edu/archives/spr2011/ entries/implicature-optimality-games.

Schwarzschild, Roger. 1991. On the Meaning of Definite Plural Noun Phrases. University of Massachusetts-Amherst PhD thesis.

Schwarzschild, Roger. 1994. Plurals, presuppositions and the sources of distributivity. Natural Language Semantics 2(3). 201-248. http://dx.doi. org/10.1007/BFo1256743.

Shannon, Claude E. 1948. A mathematical theory of communication. Bell Systems Technical Journal 27.

Sharvy, Richard. 1980. A more general theory of definite descriptions. The Philosophical Review 89(4). 607-624. http://dx.doi.org/10.2307/2184738. Sperber, Dan \& Deirdre Wilson. 1986. Relevance: Communication and Cognition. Cambridge, Mass: Blackwell, Oxford \& Harvard University Press.

Szabolcsi, Anna \& Bill Haddican. 2004. Conjunction meets negation: A study of cross-linguistic variation. Journal of Semantics 21(3). 219-250. http: //dx.doi.org/10.1093/jos/21.3.219.

Winter, Yoad. 2001. Plural predication and the strongest meaning hypothesis. Journal of Semantics 18(4). 333-365. http://dx.doi.org/10.1093/jos/18.4. 333.

Yoon, Youngeun. 1996. Total and partial predicates and the weak and strong interpretations. Natural Language Semantics 4(3). 217-236. http://dx.doi. org/10.1007/BFo0372820.

Zondervan, Arjen, Luisa Meroni \& Andrea Gualmini. 2008. Experiments on the role of the Question Under Discussion for ambiguity resolution and implicature computation in adults. Semantics and Linguistic Theory 18. 765-777. http://elanguage.net/journals/index.php/salt/issue/view/281. 
Sophia A. Malamud

Sophia A. Malamud

Department of Computer Science

Brandeis University

415 South Street, MS 018

Waltham, MA 02454-9110

smalamud@brandeis.edu 